\title{
MODELAMIENTO TERMOCINEMÁTICO 3D DE LA HISTORIA DE EXHUMACIÓN DEL SECTOR DE GUAYABETAL, KM 58 VÍA BOGOTÁ - VILLAVICENCIO: RELACIONES ENTRE CLIMA, RELIEVE Y TECTÓNICA
}

\section{D thermokinematic modeling of the exhumation history of the Guayabetal sector, km 58 vía Bogota-Villavicencio: relationships between climate, relief and tectonics}

Daniel R. Hernández Chaparroํ, Mauricio A. Bermúdez², Greg Hoke, Helbert García Delgado4, Silvia Machuca ${ }^{1}$ Universidad de Sao Paulo (USP), Instituto de Geociencias (IGC), ²Escuela de Ingeniería Geológica, Universidad Pedagógica y Tecnológica de Colombia, ${ }^{3}$ Department of Earth Sciences, Syracuse University, Syracuse, NY, USA,

${ }^{4}$ Servicio Geológico Colombiano, Bogotá, Colombia, ${ }^{5}$ Pontificia Universidad Javeriana, Bogotá, Colombia

Email: 1daniel.hernandez04@uptc.edu.co,2mauricio.bermudez@uptc.edu.co,3rgdhoke.syr.edu,

4helbertgarciad@gmail.com hsgarcia@sgc.gov.co, ${ }^{5}$ silviamachuca01@gmail.com

(Recibido el 11 de diciembre de 2020 - Aceptado el 8 de abril de 2021 )

\section{Resumen}

Las interacciones -clima-tectónica pueden dar lugar a cambios topográficos significativos tanto por aumentos en la elevación como por el incremento de pendientes. Estos cambios, en conjunción con la acción de la gravedad suscitan a su vez diferencias en la energía potencial de materiales geológicos (rocas, agua, etc.) desencadenando procesos geomórficos importantes tales como la erosión por agua, tanto laminar como concentrada, y los movimientos en masa En la vía BogotáVillavicencio en el kilómetro 58 del municipio de Guayabetal, se han presentado numerosos fenómenos de remoción en masa. La presente investigación explora como operan las interacciones entre el clima y la tectónica como procesos controladores del relieve actual a corto y largo plazo sobre la zona de estudio. Con esta finalidad se compilaron edades termocronológicas existentes en la zona, y se generó un modelo directo termocinemático 3D para estimar los pulsos y las tasas de exhumación. Los resultados de este último sugieren 3 pulsos de exhumación: el primero entre $40 \mathrm{Ma}-25 \mathrm{Ma}$ a una tasa de exhumación de 0,5 km/Ma, seguido de un pulso entre $25 \mathrm{Ma}-15 \mathrm{Ma}$ con una tasa de 0,1 km/Ma, y finalmente, desde $15 \mathrm{Ma}$ al presente tasas de $\sim 2 \mathrm{~km} / \mathrm{Ma}$. Adicionalmente, datos de precipitación fueron utilizados para estimar atributos primarios y secundarios del terreno, mientras que los datos de sismicidad instrumental fueron empleados para calcular la deformación sísmica, energía sísmica y levantamiento vertical por deformación sísmica. Las distintas variables fueron comparadas estadísticamente. Se concluye que el paisaje actual de la zona no es afectado uniformemente por la tectónica y las precipitaciones. En el noroccidente del área existen bajas tasas de erosión y actividad tectónica, así la evolución del paisaje es más pasiva, y el relieve es controlado por el patrón de precipitaciones. En contraste, hacia el suroriente específicamente entre macizo de Quetame y el piedemonte llanero, el relieve es controlado por la tectónica presente en el área. El efecto antrópico con las modificaciones al paisaje introducidas por la creación de una vía nacional, aunque es importante no fue analizado en la presente investigación.

Palabras clave: termocronología, modelamiento numérico, evolución del paisaje, Cordillera Oriental de Colombia, estabilidad de taludes, remoción en masa, geomorfometría.

\footnotetext{
Abstract

Climate-tectonic interactions can result in significant topographic changes due to both elevation and slope increases. These changes, in conjunction with the action of gravity, in turn, cause differences in the potential energy of geological materials
} 
triggering important geomorphic processes such as erosion by water, both laminar and concentrated, and mass movements. On the Bogota-Villavicencio road at kilometer 58 in the municipality of Guayabetal, numerous mass removal phenomena have occurred. This research explores how the interactions between climate and tectonics operate as controlling processes of the current short- and long-term relief over the study area. Based on this purpose, existing thermochronological ages in the area were compiled, and a direct 3D thermokinematic model was generated to estimate pulses and exhumation rates. The results of the latter suggest 3 exhumation pulses: the first one between $40 \mathrm{Ma}-25 \mathrm{Ma}$ at an exhumation rate of $0.5 \mathrm{~km} / \mathrm{Ma}$, followed by a pulse between $25 \mathrm{Ma}-15 \mathrm{Ma}$ with a rate of $0.1 \mathrm{~km} / \mathrm{Ma}$, and finally, from $15 \mathrm{Ma}$ to the present rates of $\sim 2 \mathrm{~km} / \mathrm{Ma}$. Additionally, precipitation data was used to estimate primary and secondary soil attributes, while instrumental seismicity data was used to calculate seismic deformation, seismic energy and vertical seismic deformation survey. The different variables were compared statistically. It is concluded that the current landscape of the area is not uniformly affected by tectonics and rainfall. In the northwest of the area there are low rates of erosion and tectonic activity, so the evolution of the landscape is more passive, and the relief is controlled by the pattern of precipitation. In contrast, to the southeast, specifically between the Quetame massif and the piedmont plains, the relief is controlled by the tectonics present in the area. The anthropic effect with the modifications to the landscape introduced by the creation of a national road, although it is important, it was not analyzed in this research.

Key words: thermochronology, thermal-kinematic modelling, landscape evolution, Eastern Cordillera of Colombia, slope stability, mass- wasting processes, geomorphometry.

\section{INTRODUCCIÓN}

En virtud de que posibles interacciones entre procesos climáticos y tectónicos como fenómenos de remoción en masa que ocurren en espacios con asentamientos de ciudades, pueblos y caseríos, podrían ocasionar daños materiales y pérdidas humanas, el estudio de zonas tectónicamente activas, y en particular del sistema montañoso andino, adquiere relevancia desde la perspectiva de la ingeniería geológica o geología aplicada debido a la complejidad geológica de este sistema.

La presente investigación explora la forma que operan las interacciones entre el patrón de precipitaciones actuales y la tectónica como procesos controladores del relieve actual a corto y largo plazo, integrando los distintos factores mostrados en la Figura 1. El área de estudio se encuentra en los alrededores del km 58 de la vía Bogotá-Villavicencio (ver triángulo rojo en la Figura 2), Cordillera Oriental de Colombia, tomando como base el modelamiento numérico de datos termocronológicos existentes, en particular edades de trazas de fisión en apatitos (por sus siglas en inglés: Apatite Fission-Track, AFT) y trazas de fisión en circón (por sus siglas en inglés: \}
Zircon Fission-Track, ZFT), parámetros geomorfométricos, datos sismológicos y litología. Para ello se siguió la metodología propuesta por Bermúdez et al. [1, 2, 3], quienes combinaron datos geológicos recolectados en campo con métodos de termocronología cuantitativa [4, $5,6]$, con lo que se desarrolló el modelado de la historia de exhumación de al menos 35 superficies de erosión del altiplano antioqueño en Colombia, discriminando en cada una de esas superficies los posibles controles tectónicos, climáticos o la interacción de ambos como agentes rejuvenecedores del paisaje actual, lo cual constituyó la primera aplicación del software PECUBE ${ }^{\circledR}$ en Colombia.

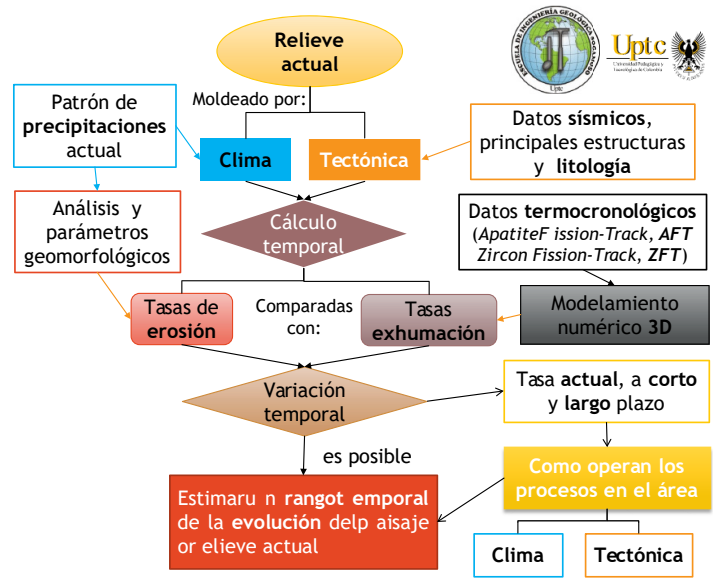


Daniel R. Hernández Chaparro

Mauricio A. Bermúdez

Greg Hoke

Helbert García Delgado

Silvia Machuca

Figura 1. Esquema de los componentes para el análisis del relieve actual.

\subsection{Localización}

Se observa en la Figura 2, el Valle del Río Negro (también llamado Río Guayuriba), caracterizado por la presencia de un valle ancho con relieve ondulado de clima frío, con topografía más abrupta hacia el $\mathrm{S}$ en los dominios del Macizo de Quetame, este valle muy densamente poblado incluye en sus laderas a las poblaciones de Ubaque, Fómeque, Cáqueza, Quetame y Fosca; al W de este valle a están los municipios de Une y Chipaque, al oriente en el cañón del Río Guatiquia los municipios de San Juanito y El Calvario. El Río Negro continua hacia el S hasta Guayabetal, donde se ubica el deslizamiento del Km 58, objeto de esta investigación, luego del municipio el Río Negro se une con el Río Blanco cambiando su nombre a Río Guayuriba; el valle del Río Blanco marca una franja al $\mathrm{S}$ del área de estudio, además se encuentran poblaciones del departamento del Meta como Acacias y Villavicencio.

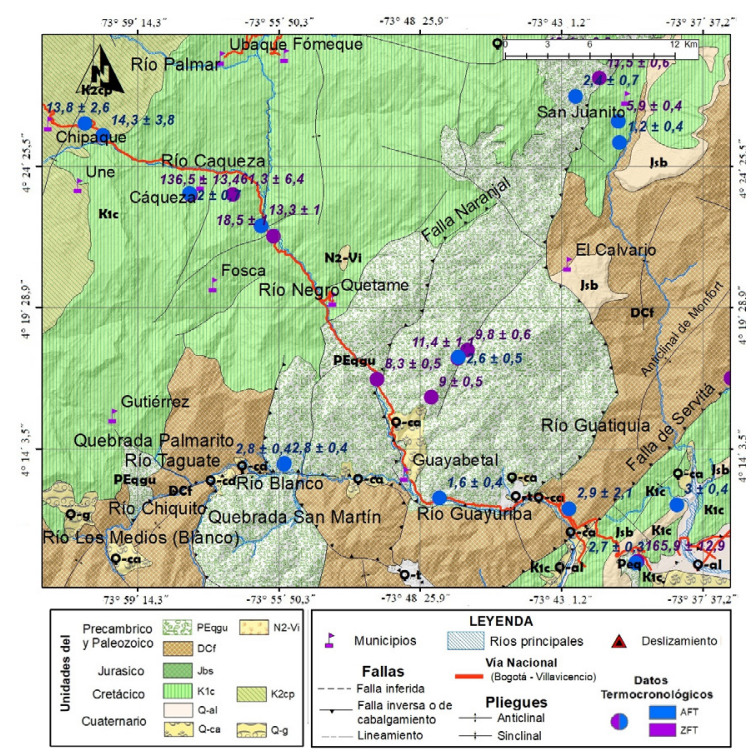

Figura 2. Mapa geológico [7] que comprende la vía BogotáVillavicencio y distribución de edades termocronológicas existentes [8,9], se denota sobre este mapa en triángulo rojo la zona de deslizamientos del km-58, los puntos azules y morados corresponden con la ubicación de muestras previamente fechadas por los métodos de trazas de fisión en apatitos (AFT) y circones (ZFT), respectivamente.

\subsection{Antecedentes}

Históricamente, la autopista al Llano ha sido escenario de múltiples accidentes automovilísticos y derrumbes, debido a las difíciles condiciones topográficas de la Cordillera Oriental, y también a errores de ingeniería. El más recordado de estos sucesos es conocido como la tragedia de Quebrada Blanca ocurrida el 28 de junio de 1974 que dejó un número significante de personas fallecidas.

El trazado de la vía comienza justo después de salir de Bogotá, se encuentra el túnel Argelino Durán Quintero, también conocido como túnel del Boquerón, de 2,2 km de longitud, en cuya salida se ubica el peaje de Boquerón. Tras esto, se encuentran el municipio de Chipaque, y los accesos a Une y Cáqueza. Poco antes de llegar a Quetame, la vía toma un trayecto paralelo al Río Negro a lo largo del valle del mismo, lo que la hace susceptible a verse afectada por derrumbes de tierra. Entre Quetame y Guayabetal, se encuentran el peaje de Naranjal y el túnel de Quebrada Blanca, construido tras la tragedia ocurrida en 1974. Después de Guayabetal se encuentran los sectores de Chirajara y Pipiral.

\subsubsection{Deslizamiento del $\mathrm{km} 58$, Bogotá - Villavicencio.} Se presenta el cierre exactamente entre los kilómetros 57 y 58,5 en la zona denominada de Mesa Grande el 14 de junio del 2019, que conllevaron al derrumbe de más de $600.000 \mathrm{~m}^{3}$ de material no consolidado, de los cuales $225.000 \mathrm{~m}^{3}$ generaron la obstaculización total de la vía (Figura 3), cerca de la población de Guayabetal (Cundinamarca) siendo ésta la más afectada, lo que conllevó al Ministerio del Transporte tomar una medida de suspensión de transitabilidad de forma indefinida.

Este fenómeno de remoción en masa en una terraza coluvio-aluvial colgada sobre filitas (PEqgu; Figura 2); corresponde a un deslizamiento de tipo complejo traslacional con reactivación latente de flujo de detritos y desprendimiento de talud activado por las altas precipitaciones de la época de lluvias. 


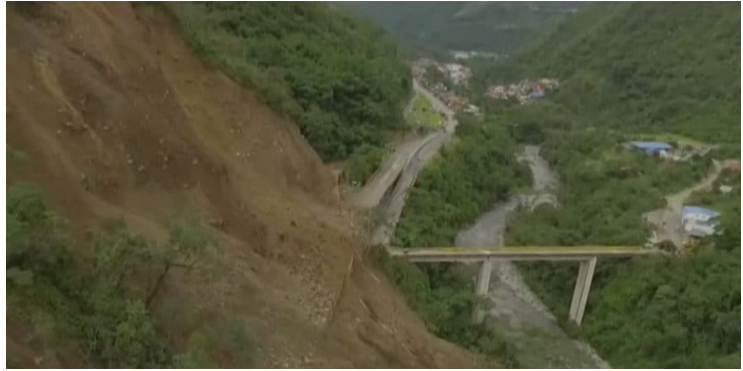

Figura 3. Derrumbe en vía Bogotá-Villavicencio; (Fuente: Captura video Noticias Caracol).

\section{MARCO GEOLÓGICO}

La Cordillera Oriental de Colombia es la tercera y la más joven de las tres cordilleras andinas en Colombia, siendo ésta la última en exhumarse como resultado de una interacción compleja entre las placas Caribe, Nazca-Cocos, Suramérica y acreciones de bloques [10]. Según Mora [11], al igual que muchos otros autores, esta cordillera corresponde a un orógeno que se invirtió durante una fase compresiva cenozoica, valiéndose de estructuras reactivadas de edad mesozoicas producidas durante una fase de rift. Entre esas importantes estructuras reactivadas que hoy en día producen la carga tectónica y el acortamiento de la montaña, están las fallas Servitá, San Juanito y Naranjal, situadas en el flanco oriental del orógeno (Figura 2 y 4 ).

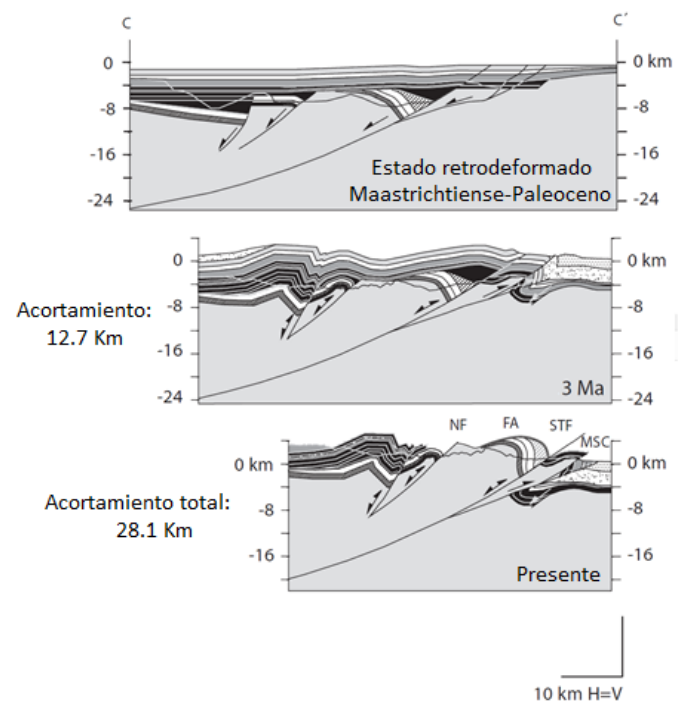

Figura 4. Perfil de la sección $C-C^{\prime}$, Reconstrucción de historia estructural de la retrodeformación basada en datos termocronológicos, reflectancia de vitrinita Ro, datos sísmicos y de paleoaltimetría (paleoflora). Abreviaciones: NF-Falla de Naranjal; FA - anticlinal de Farallones; MSC - Falla de Mirador; STF_Falla de Servitá (Modificado de [8])

La falla de Naranjal se generó durante el Mesozoico por tectónica extensional y durante el Neógeno sufrió una reactivación, cambiando el mecanismo de falla normal a inversa. (ver Figura 16, Perfil de Río Guayuriba [11]), hoy en día presenta una expresión moderada localizada en el flanco oriental de la Cordillera Oriental entre las latitudes 3,6 y $4,5^{\circ} \mathrm{N}$ (Figura 4), esta falla yace en el bloque colgante de la Falla de Servitá, la cual es muy activa. A la Falla de Naranjal se le han asociado los destructores sismos de Quetame del 2008 (evento de magnitud 5.7 y profundidad de 300 m ocurrido el 24 de mayo del 2008) y Fómeque de 1743 (evento ocurrido el 18 de octubre de 1743 de magnitud macrosísmica igual a $6.30 \pm 0.35$ calculada aplicando la metodología de Bakun y Wentworth [12] y la relación de atenuación de la intensidad macrosísmica [13].

Cabe resaltar la cercanía de la falla activa de Naranjal con el deslizamiento del km 58 es un deslizamiento complejo sobre una terraza alta disectada de un depósito aluvial reciente (Q-ca en Figura 2) de sobre unas filitas fracturadas al costado oriental del Río Negro (Río Guayuriba), a lo largo de este último son comunes los depósitos aluviales, formados principalmente a lo largo de los cauces de los ríos, los cuales son el resultado de la acumulación de sedimentos gruesos a muy gruesos en las zonas de menor energía, conformando terrazas angostas y alargadas, compuestas esencialmente de gravas, guijos y cantos en una matriz areno-lodosa no consolidada, los cuales son producto, además, de la tectónica activa en las fallas de piedemonte que acentúan los procesos de erosión y sedimentación hacia las zonas bajas, sobre todo en la época invernal.

Este depósito aluvial reciente (Q-ca) con baja consolidación amplifica la onda sísmica liberada por la falla de Naranjal al 
transferir energía sísmica de eventos sísmicos superiores a 4 en la escala de Richter, posiblemente, influenciando de manera directa o indirecta un fenómeno de remoción en masa siendo la amenaza sísmica el factor detonante o condicionante debido al alto potencial sismogénico de esta falla.

En el centro del área de estudio afloran rocas metamórficas, metasedimentarias como el Grupo Esquistos de Quetame (Peq) y su formación Filitas y Cuarcitas de Guayabetal (PEqgu) y sedimentarias del Precámbrico y Paleozoico refiriéndose al Grupo Farallones (DCf) aflorante en gran proporción en el S-E del área de estudio; las cuales se presentan discordantes entre si y constituyen el basamento del área. De igual forma, reposan discordantemente las unidades del Jurásico Superior como la Formación Brechas de Buenavista (Jsb) y del Cretácico inferior como el grupo Cáqueza (K1c) y la formación Chipaque (K2cp) aflorantes en la zona $\mathrm{N}-\mathrm{W}$ del área de estudio; los sedimentos cuaternarios no consolidados están constituidos por varios niveles de terrazas, conos o abanicos aluviales, depósitos de ladera o coluviones y sedimentos aluviales recientes. (INGEOMINAS, 2011).

Se presenta el flanco oriental del Sinclinal de Teusacá y en el centro del área de estudio el Anticlinal de Potrero Grande desde el municipio de Fomeque (Figura 5) hasta Quetame en donde se ve cortado por la Falla de Quetame, siendo las principales estructuras además de la Falla de San Juanito la cual separa un bloque occidental cuyo basamento Infra-Cretácico lo constituye el Grupo Quetame, del bloque oriental, unidad infrayacente al Cretácico basal es la Formación Capas Rojas del Valle de Guatiquia de Paleozoico Superior [4].

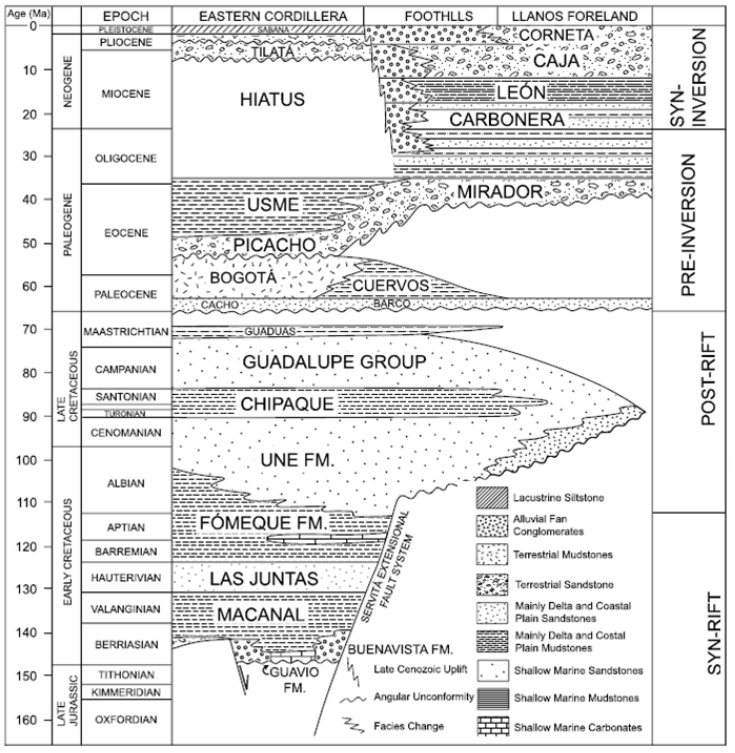

Figura 5. Estratigrafía meso-cenozoica del flanco oriental de la Cordillera Oriental (Río Guayuriba - Guatiquía) Tomada de: Mora et al., 2006.

\section{BASES DE DATOS Y MÉTODOS}

En la Figura 6, se muestran en los cuadros rellenos de color las bases de datos utilizadas en la presente investigación, mientras en los cuadros blancos se presenta la referencia de extracción de los datos.

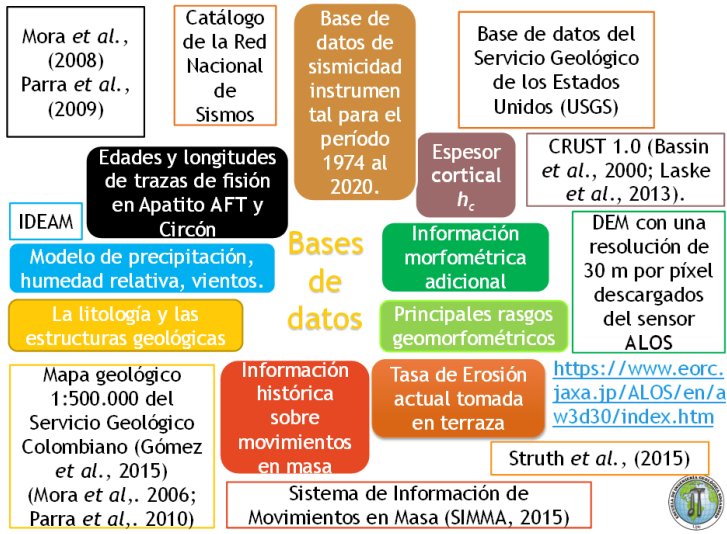

Figura 6. Bases de datos usada en esta investigación.

Las unidades litológicas de las cuales se tienen datos termocronológicos se resumen en la Tabla 1. 
Tabla 1. Principales Unidades Litológicas aflorantes en el área de estudio [14, 15, 16. 17. 18].

\begin{tabular}{|c|c|c|c|}
\hline Símbolo & $\begin{array}{l}\text { Grupos y } \\
\text { unidades }\end{array}$ & Descripción litológica & Edad \\
\hline $\mathrm{K} 2 \mathrm{cp}$ & $\begin{array}{l}\text { Formación } \\
\text { Chipaque }\end{array}$ & $\begin{array}{c}\text { Shales, calizas, } \\
\text { fosforitas, cherts } \\
\text { y cuarzoarenitas. } \\
\text { Predominio de facies } \\
\text { finas al norte del Cocuy } \\
\text { y facies más arenosas } \\
\text { al sur. }\end{array}$ & $\begin{array}{l}\text { Cenomaniano- } \\
\text { Maastrichtiano }\end{array}$ \\
\hline \multirow[b]{2}{*}{$\mathrm{K} 1 \mathrm{C}$} & $\begin{array}{l}\text { Formación } \\
\text { Fomeque }\end{array}$ & $\begin{array}{c}\text { Alternancia de } \\
\text { conjuntos de lutitas } \\
\text { negras y conjuntos } \\
\text { de calizas y areniscas } \\
\text { calcáreas }\end{array}$ & \multirow[b]{2}{*}{$\begin{array}{c}\text { Berriasiano- } \\
\text { Cenomaniano }\end{array}$} \\
\hline & $\begin{array}{l}\text { Grupo } \\
\text { Cáqueza }\end{array}$ & $\begin{array}{c}\text { Arcillolitas y } \\
\text { limolitas negras } \\
\text { con intercalaciones } \\
\text { menores de arenitas } \\
\text { y calizas. Segmentos } \\
\text { de cuarzoarenitas de } \\
\text { grano fino a grueso y } \\
\text { conglomerados. }\end{array}$ & \\
\hline Jsb & $\begin{array}{l}\text { Formación } \\
\text { Brechas de } \\
\text { Buenavista }\end{array}$ & $\begin{array}{l}\text { Conglomerados y } \\
\text { brechas; arenitas } \\
\text { de grano fino a } \\
\text { conglomeráticas y } \\
\text { calizas estromatolíticas, } \\
\text { e intercalaciones de } \\
\text { lodolitas negras. }\end{array}$ & Titoniano \\
\hline DCf & $\begin{array}{l}\text { Grupo } \\
\text { Farallones }\end{array}$ & $\begin{array}{c}\text { Cuarzoarenitas, } \\
\text { arcillolitas, } \\
\text { lodolitas grises y, } \\
\text { ocasionalmente, calizas } \\
\text { y conglomerados. }\end{array}$ & $\begin{array}{l}\text { Devónico- } \\
\text { Carbonífero }\end{array}$ \\
\hline PEqgu & $\begin{array}{c}\text { Filitas y } \\
\text { Cuarcitas } \\
\text { de } \\
\text { Guayabetal }\end{array}$ & $\begin{array}{c}\text { Filitas verdosas y } \\
\text { cuarcitas }\end{array}$ & $\begin{array}{l}\text { Ordovícico- } \\
\text { Llandovery }\end{array}$ \\
\hline
\end{tabular}

\subsection{Bases de datos termocronológicas}

En estas formaciones fueron tomados 25 datos termocronológicos de edades AFT y ZFT [8, 9], los cuales fueron usados en este trabajo para la modelización numérica (Tabla 2).

Adicionalmente, se compilaron 7 edades y tasas de incisión fluvial mediante isótopos cosmogénicos (Berilio-10) realizadas en terrazas del Río Guayuriba [19], los cuales fueron interpolados a lo largo de la zona de estudio usando el método IDW de la licencia académica existente en la Universidad de $\operatorname{ArcGIS}{ }^{\circledR}$.
Tabla 2. Edades termocronológicas existentes en la zona de estudio: Edades por trazas de fisión en apatito (AFT) y circón (ZFT) [8,9]. En la Figura 1, se ubica cada muestra por su código en el área de estudio.

\begin{tabular}{|c|c|c|c|c|c|c|c|}
\hline Código & Latitud & Longitud & $\begin{array}{c}\text { Unidad o } \\
\text { Grupo }\end{array}$ & AFT & $\begin{array}{c}\text { error } \\
\text { AFT }\end{array}$ & ZFT & $\begin{array}{c}\text { error } \\
\text { ZFT }\end{array}$ \\
\hline $\begin{array}{l}\text { BV- } \\
\text { MP3-F }\end{array}$ & 44.427 & -740.207 & & 13,8 & 2,6 & N/A & 0 \\
\hline $\begin{array}{l}\text { BV- } \\
\text { MP7-F }\end{array}$ & 44.349 & -738.003 & $\begin{array}{c}\text { F. } \\
\text { Chipaque }\end{array}$ & 14,3 & 3,8 & N/A & 0 \\
\hline SJ-2a & 44.304 & -740.094 & & 1,2 & 0,4 & N/A & 0 \\
\hline $\begin{array}{c}\text { BV } \\
120-F\end{array}$ & 43.979 & -736.802 & & 2 & 0,7 & N/A & 0 \\
\hline BV-151 & 43.705 & -739.543 & & 3,8 & 0,7 & 18,5 & 1 \\
\hline BV126 & 43.772 & -739.008 & G. Cáqueza & N/A & 0 & 13,3 & 1 \\
\hline BV-65 & 43.970 & -739.087 & & N/A & 0 & 61,3 & 6,4 \\
\hline SJ-1 & 44.438 & -739.266 & & N/A & 0 & 5,9 & 0,4 \\
\hline BV-121 & 43.968 & -736.809 & & N/A & 0 & 136,5 & 13,4 \\
\hline BV-427 & 41.986 & -739.533 & $\begin{array}{l}\text { Formación } \\
\text { Brechas de } \\
\text { Buenavista }\end{array}$ & 3,0 & 0,4 & N/A & 0 \\
\hline WPT & 41.962 & -736.438 & G. & 2,9 & 2,1 & N/A & 0 \\
\hline BV-283 & 42.796 & -737.124 & Farallones & N/A & 0 & 145,2 & 17,3 \\
\hline BV-192 & 42.674 & -736.091 & & 2,3 & 0,6 & 9 & 0,5 \\
\hline BV-195 & 42.933 & -738.003 & & 2,6 & 0,5 & N/A & 0 \\
\hline BV-196 & 42.925 & -737.828 & & 2,6 & 1,1 & 11,4 & 1,1 \\
\hline BV-277 & 41.614 & -737.834 & & 2,7 & 0,3 & $\mathrm{~N} / \mathrm{A}$ & 0 \\
\hline BV-279 & 41.624 & -736.702 & & 2,6 & 0,3 & 165,9 & 12,9 \\
\hline FT-1 & 44.889 & -736.690 & & N/A & 0 & 13,1 & 0,9 \\
\hline SJ-5 & 44.716 & -737.312 & & N/A & 0 & 11,5 & 0,6 \\
\hline BV-423 & 42.029 & -736.931 & Filitas y & 1,6 & 0,4 & $\mathrm{~N} / \mathrm{A}$ & 0 \\
\hline BV-90 & 42.245 & -737.951 & $\begin{array}{c}\text { de } \\
\text { Guayabetal }\end{array}$ & 2,8 & 0,4 & N/A & 0 \\
\hline BV-86 & 42.789 & -738.941 & & N/A & 0 & 8,3 & 0,5 \\
\hline BV-194 & 42.976 & -738.346 & & N/A & 0 & 9,8 & 0,6 \\
\hline BV-90 & 42.251 & -737.771 & & 2,8 & 0,4 & N/A & 0 \\
\hline FT-6 & 44.599 & -738.938 & & 2,4 & 0,7 & N/A & 0 \\
\hline
\end{tabular}

3.1.1. Índice de erosión a largo plazo. Las distribuciones de las edades termocronológicas correspondientes a edades AFT y ZFT se utilizaron para calcular las tasas de exhumación o de erosión a largo plazo, asumiendo que la cantidad de erosión es igual al levantamiento, esto equivale a decir que la topografía se encuentra en estado de equilibrio [20]. Con este propósito se empleó 
la rutina Age2Edot desarrollada por Brandon [21]. Los parámetros de entrada de esta rutina son: 1) el tipo de mineral fechado (apatito o circón), 2) el método utilizado (trazas de fisión), 3) la difusividad termal, habiendo en este caso usado $25 \mathrm{~km}^{2} \mathrm{My}^{-1}$, 4) el espesor del modelo de $40 \mathrm{~km}$ basado en el espesor cortical promedio del modelo CRUST1.0 [22, 23] usado, y 5) una temperatura en la base del modelo fija de $400{ }^{\circ} \mathrm{C}$, al igual que la considerada para los modelos termocinemáticos. Esto permitió convertir cada edad termocronológica en una tasa de erosión que es comparada con los otros métodos.

\subsubsection{Modelamiento termocinemático tridimensional.} Con el fin de establecer la historia de exhumación de este sector de la Cordillera Oriental, se utilizaron datos termocronológicos AFT y ZFT existentes (Tabla 2) $[11,9]$, se utilizó el DEM, y los parámetros resumidos en la Tabla 2. Todos estos fueron incorporados al código termocinemático 3D PECUBE en su versión directa ("forward") desarrollado por Braun $[4,5,6]$ el cual permite integrar distintos parámetros termales, flexurales, topográficos y estructurales (orientaciones y geometrías de fallas). El código resuelve la ecuación del calor en tres dimensiones, permitiendo predecir edades termocronológicas para distintos sistemas, y elevaciones. De estas predicciones, fue posible generar perfiles edadelevación sintéticos, estimar tasas de erosión a largo plazo; además de estudiar la influencia de zonas de fallas en la región analizada.

Los parámetros texturales y flexurales restringen la respuesta termal del área modelada a cambios de exhumación y relieve. La historia termal de puntos en la superficie se usa para predecir las edades de AFT y ZFT tomando en cuenta la temperatura de control sobre el desvanecimiento de las huellas "annealing track" [24].

Se realizó un total de 10000 modelos directos "forward" asumiendo tres y cuatro fases de exhumación. Se asume el periodo inicial del modelo y algunos parámetros flexurales y termales fijos como datos de entrada del código, los cuales son mostrados en la Tabla 3.
Tabla 3. Parámetros de entrada fijos, las filas sin color de relleno se refieren a los parámetros flexurales, mientras que aquellas de color gris corresponden con las variables termales.

\begin{tabular}{ccc}
\hline Parámetro & Valor & Referencias \\
\hline Tiempo inicial del modelo & $40(\mathrm{Ma})$ & \\
Densidad de la corteza & $2700\left(\mathrm{~kg} / \mathrm{m}^{3}\right)$ & {$[25]$} \\
Densidad de la astenosfera & $3200\left(\mathrm{~kg} / \mathrm{m}^{3}\right)$ & {$[25]$} \\
Módulo de Young & $1 . \mathrm{d} 11$ & {$[25]$} \\
Coeficiente de Poisson & 0,25 & {$[25]$} \\
Espesor del modelo & $40(\mathrm{~km})$ & {$[22,23]$} \\
Difusividad termal & $25(\mathrm{~km} / \mathrm{Ma})$ & {$[26]$} \\
Temperatura en la base del & $400\left({ }^{\circ} \mathrm{C}\right)$ & {$[1]$} \\
modelo & $22\left({ }^{\circ} \mathrm{C}\right)$ & \\
Temperatura a nivel del mar & & \\
\hline
\end{tabular}

Con estos parámetros termales y flexurales de entrada, se restringe la respuesta termal a cambios en la exhumación y relieve (Figura 6), luego se busca la obtención del menor ajuste entre edades observadas y predichas ("misfit"). Por esta razón, se computan diversos modelos forward para abarcar una amplia gama de posibles soluciones en diferentes escenarios, predominantemente, bajo el control de datos estructurales, litológicos y geotermales existentes en la literatura. La selección del mejor modelo se basa en la minimización del error cuadrático medio o 'misfit' en la Ecuación 1.

$$
\text { Misfit }=\frac{1}{n} \sqrt{\frac{\sum_{i=1}^{n}\left(t_{o b s}^{i}-t_{c a l}^{i}\right)^{2}}{\text { error } r_{o b s}^{i}}}
$$

Donde $n$ es el número de observaciones, tobs y tcal corresponde a las edades observadas y predichas por PECUBE para cada una de las muestras $i$.

\subsection{Precipitación}

Los registros meteorológicos de precipitación, humedad y viento durante el período 1990- 2019 fueron provistos por el Instituto de Hidrología, Meteorología y Estudios Ambientales de Colombia (IDEAM). En la Tabla 4, se presentan las estaciones meteorológicas utilizadas y los valores promedios en el intervalo de tiempo antes 
mencionado. La precipitación mínima media encontrada fue de $12 \mathrm{~mm} / \mathrm{mes}$ en la estación de "Casas Las", mientras la máxima corresponde a 1040,1 mm/mes encontrada en la estación de Buenavista para el mes de junio. Estos valores fueron interpolados en ArcGIS usando el método de distancia inversa ponderada, en el cual se determinan los valores de celda a través de una combinación lineal ponderada de un conjunto de datos de precipitaciones [27], presentando el modelo de precipitaciones anual en la Figura 7. Según el análisis del clima en la cuenca del Río Guayuriba del "POMCA (Plan de Manejo y Ordenamiento de una Cuenca) Guayuriba" [28] la dirección predominante del viento en 3 de las estaciones meteorológicas (Monteredondo, Servitá y Susumuco; Tabla 3) en todos los meses es desde el norte y el noreste, si bien en el segundo trimestre del año se tiene una mayor proporción de vientos que corren desde esta dirección. Se destaca también que en el trimestre de junio a agosto se incremente la proporción de viento que viene desde el oeste y el noroeste; esto puede estar asociado de cierta manera a la temporada de lluvias, pues los eventos de precipitación en el piedemonte pueden generar vientos a escala local, cerca de la superficie.

Tabla 4. Valores promedios mensuales de precipitación del periodo 1990-2019 para algunas estaciones meteorológicas del IDEAM cercanas a la zona de estudio.

\begin{tabular}{|c|c|c|c|c|c|c|c|c|c|c|}
\hline Estación & Gutiérrez & Servita & $\begin{array}{c}\text { El } \\
\text { Calvario }\end{array}$ & Monfort & Fómeque & $\begin{array}{l}\text { Buena- } \\
\text { Vista }\end{array}$ & $\begin{array}{l}\text { Monter- } \\
\text { Redondo }\end{array}$ & $\begin{array}{l}\text { Susu- } \\
\text { Muco }\end{array}$ & Casas Las & Llano Largo \\
\hline Código & 35020300 & 35030291 & 35030010 & 35030021 & 35020290 & 35030091 & 35020011 & 35020020 & 35030080 & 35025051 \\
\hline$x$ & 1008305 & 1042677 & 1040794 & 1047675 & 1020763 & 1051029 & 1028217 & 1033927 & 1015662 & 1005241 \\
\hline Y & 962150,4 & 954960,4 & 972784,5 & 968374,3 & 987874,9 & 964472,0 & 962213,0 & 955770,6 & 982857,7 & 987463,9 \\
\hline Altitud & 2300,0 & 191,0 & 1800,0 & 1100,0 & 1900,0 & 1280,0 & 1300,0 & 10,0 & 2100,0 & 2980,0 \\
\hline Enero & 70,0 & 393,9 & 91,0 & 229,2 & 38,4 & 508,6 & 97,6 & 217,4 & 34,0 & 67,2 \\
\hline Febrero & 69,9 & 109,9 & 30,0 & 94,6 & 16,2 & 164,5 & 41,1 & 81,2 & 12,0 & 20,7 \\
\hline Marzo & 84,4 & 132,0 & 61,0 & 167,9 & 29,0 & 164,7 & 69,7 & 86,9 & 27,1 & 56,4 \\
\hline Abril & 128,1 & 478,5 & 161,6 & 341,3 & 71,8 & 533,9 & 167,0 & 312,6 & 67,2 & 112,2 \\
\hline Mayo & 226,6 & 764,4 & 292,0 & 639,6 & 120,6 & 898,9 & 264,7 & 505,2 & 133,6 & 176,5 \\
\hline Junio & 316,1 & 922,6 & 378,3 & 927,2 & 159,1 & 1040,1 & 374,2 & 708,6 & 129,8 & 187,5 \\
\hline Julio & 358,3 & 811,5 & 398,1 & 768,3 & 123,3 & 933,1 & 354,7 & 638,9 & 99,4 & 203,2 \\
\hline Agosto & 259,0 & 742,7 & 444,2 & 726,4 & 124,9 & 953,0 & 414,1 & 647,3 & 114,4 & 209,5 \\
\hline Septiembre & 252,1 & 619,0 & 360,0 & 636,2 & 107,8 & 688,0 & 346,0 & 575,5 & 84,5 & 152,1 \\
\hline Octubre & 149,0 & 567,2 & 271,3 & 632,2 & 68,4 & 636,8 & 230,5 & 455,4 & 72,2 & 71,1 \\
\hline Noviembre & 131,6 & 598,5 & 198,8 & 590,1 & 100,6 & 789,5 & 191,0 & 326,8 & 66,6 & 114,3 \\
\hline Diciembre & 132,6 & 707,1 & 151,9 & 404,3 & 97,8 & 837,6 & 132,5 & 308,1 & 81,1 & 140,2 \\
\hline Prec Anual & 2177 & 6847 & 2839 & 6157 & 1058 & 8149 & 2683 & 4864 & 922 & 1511 \\
\hline $\begin{array}{l}\text { Evapotrans } \\
\text { piración }\end{array}$ & 1293 & 1389 & 1324 & 1422 & 889 & 1366 & 1002 & 1648 & 753 & 742 \\
\hline $\begin{array}{l}\text { Humedad } \\
\text { relativa }\end{array}$ & 84 & 89 & 90 & 90 & 74 & 79 & 80 & 89 & 62 & 61 \\
\hline
\end{tabular}


Daniel R. Hernández Chaparro Mauricio A. Bermúdez Greg Hoke Helbert García Delgado Silvia Machuca

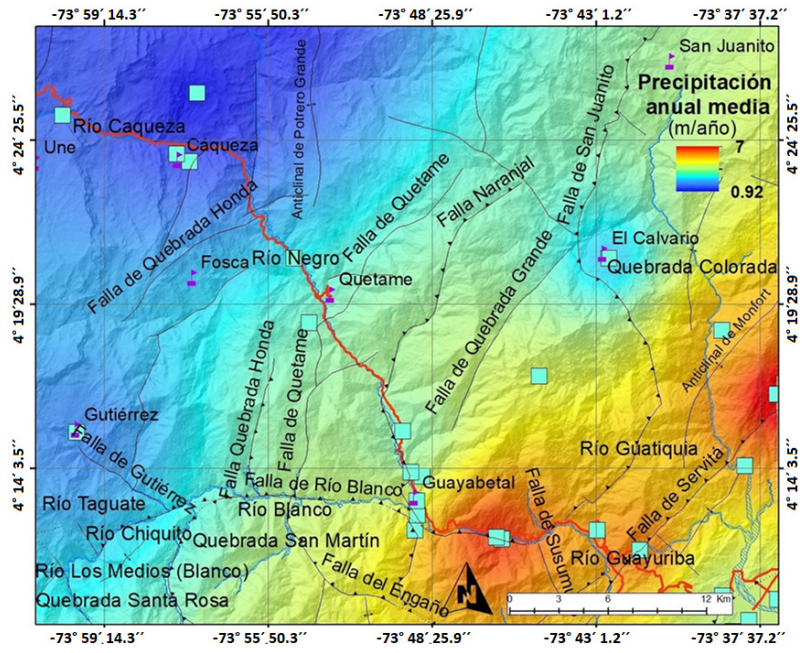

Figura 7. Mapa de precipitaciones promedios (m/año) generado mediante interpolación tipo kriging de ArcGIS a partir de los datos del IDEAM para el periodo 1990 a 2019. En esta figura, los cuadros en azul claro corresponden a las estaciones climáticas de donde se extrajeron los datos, sobre este mismo se han colocado las principales fallas geológicas de las zonas de estudio (Tomadas [7]). La ubicación de las estaciones puede ser detalladas en la Tabla 1, en esta figura el triángulo rojo corresponde al deslizamiento objetivo.

\subsection{Sismicidad}

A partir de la base de datos del Servicio Geológico de los Estados Unidos (USGS por sus siglas en inglés) y del catálogo de la Red Sismológica Nacional-RSN- del Servicio Geológico Colombiano, se compiló una base de datos de sismicidad instrumental para el período 1974 al 2020. A partir de esta última, se construyó el mapa mostrado en la Figura 3, y se calcularon distintos parámetros sísmicos como: deformación (DS), energía (ES) y levantamiento (U), con los parámetros flexurales de entrada resumidos en la Tabla 6. Cabe acotar que sólo se consideraron los sismos con profundidad menor o igual a $15 \mathrm{~km}$ [3] con el fin de analizar el aporte de estos a la deformación vertical.

Se detalla en la Figura 8, los principales eventos sísmicos en la zona, que ocurrieron en Fómeque el 18 de octubre de 1734 y en El Calvario el 24 de mayo del 2008, cuyas magnitudes fueron de 6,3 y 5,7 en la escala de Richter respectivamente. El evento más reciente, con $\mathrm{Mw}$ 6,0, localizado en las coordenadas 4,454 grados latitud Norte y 73,635 longitud Oeste, a una profundidad de 3,9 km, generó diversos deslizamientos en la vía nacional BogotáVillavicencio, donde hubo pérdidas humanas en el año 2008 durante el bloqueo de la vía [29].

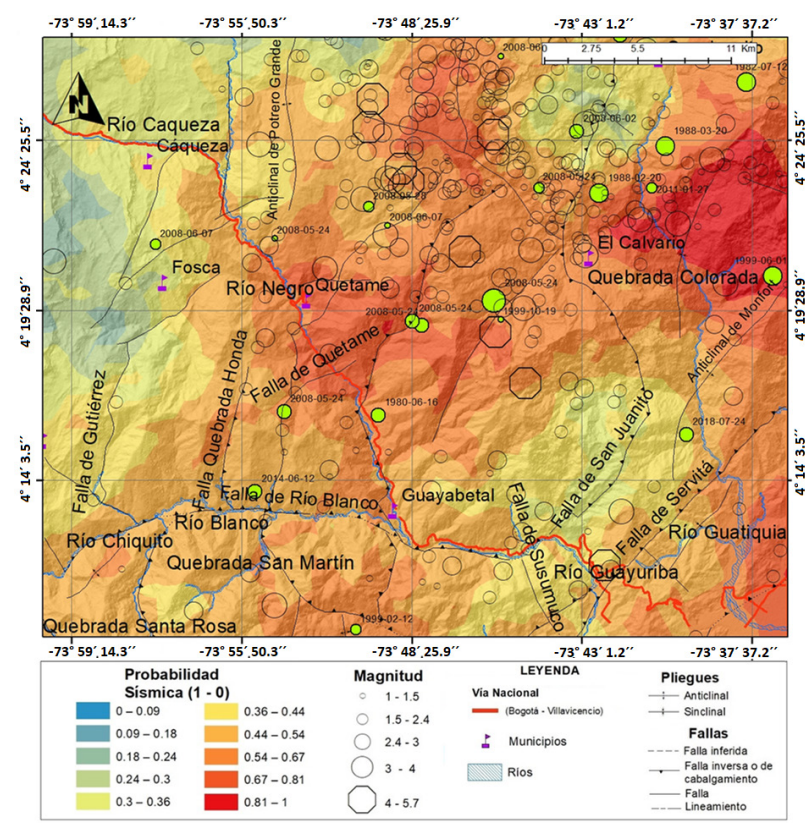

Figura 8. Mapa de sismicidad del sector estudiado, basado en los catálogos del Servicio Geológico de los Estados Unidos (USGS por sus siglas en inglés), de sismicidad histórica, y la red nacional de sismos del Servicio Geológico Colombiano entre 1974 y 2020, reportados sobre mapa estructural del [7]. Igualmente, reporta la probabilidad sísmica en escala de colores, realizada por cokriging interpolando magnitud y profundidad en ArcGIS.

\subsection{División en bloques litotectónicos}

En la Figura 9, se presenta la delimitación litotectónica realizada de acuerdo con las fallas presentes en la zona de estudio e información estructural obtenida del perfil geológico provisto por [9]. El perfil antes mencionado se construyó tomando como base las líneas sísmicas de la Agencia Nacional de Hidrocarburos (ANH), siendo posible comparar por sectores los distintos parámetros obtenidos en cada uno de los once dominios litotectónicos discriminados en esta investigación. 


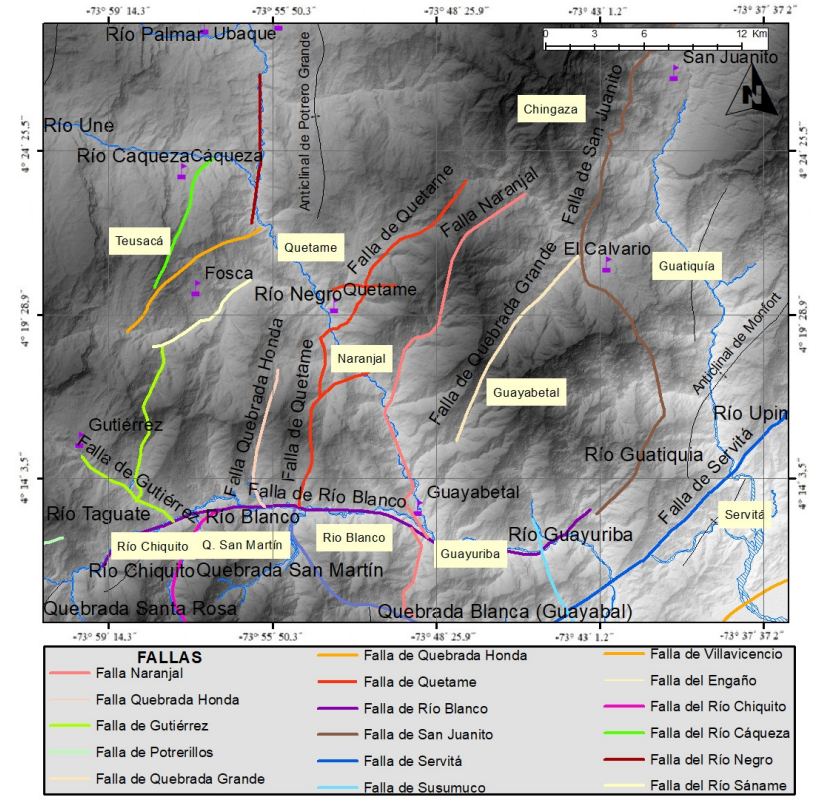

Figura 9. Delimitación de bloques litotectónicos (en fondo amarillo) propuestos entre las principales fallas en colores, tomadas del mapa geológico-estructural del SGC [7], donde la línea roja corresponde a la Vía Bogotá a Villavicencio S-E y los triángulos corresponden a los principales fenómenos de remoción en masa tomados del catálogo electrónico del Sistema de Información de Movimientos en Masa [30].

Posteriormente, empleando el modelo de elevación digital y la base de datos de precipitaciones de cada uno de los bloques litotectónicos, se calcularon los siguientes atributos primarios y secundarios del terreno: Relieve local a 1 y 2,3 km; integral hipsométrica (HI); capacidad de transporte de sedimentos (STI); índice topográfico de humedad (WI); índices de erosión: poder erosivo total (TSP, Total Stream Power), poder erosivo por cizalla SSP (Shear Stress Power) y poder erosivo unitario USP (Unit Stream Power); índice de gradiente longitudinal del canal (SL) e índice de empinamiento (ksn). Las ecuaciones para cada uno de estos parámetros se resumen en la Tabla 5.

Tabla 5. Variables morfométricas, atributos primarios y secundarios del terreno calculados para la zona de estudio, a partir del modelo de elevación digital y la base de datos de precipitaciones.

\begin{tabular}{|c|c|c|c|}
\hline Parámetro & Ecuación & & Referencia \\
\hline $\begin{array}{l}\text { Relieve local } \\
\text { (R) }\end{array}$ & $\begin{array}{c}R=D E M-\min (D E M) \\
\text { Calculado usando radios de } 1 \text { y } 2,3 \mathrm{~km} \\
\text { en ArcGIS }\end{array}$ & (2) & [31] \\
\hline $\begin{array}{l}\text { Integral } \\
\text { hipsométrica } \\
\text { (HI) }\end{array}$ & $H s i=\frac{\text { Elevación promedio }- \text { Elevación minima }}{\text { Elevación maxima }- \text { Elevación minima }}$ & (3) & [32] \\
\hline $\begin{array}{l}\text { Capacidad de } \\
\text { transporte de } \\
\text { sedimentos } \\
\text { (STI) }\end{array}$ & $\begin{array}{c}S T I=(m+1) \times(A s / 22.13)^{m} \times \sin (S / 0.0896)^{n} \\
\text { As es el área de acumulación de flujo, y } S \\
\text { es la pendiente. En esta investigación se } \\
\text { asumió que } m=0,4 ; n=1 .\end{array}$ & (4) & {$[33,34]$} \\
\hline $\begin{array}{c}\text { Índice } \\
\text { topográfico } \\
\text { de humedad } \\
\text { (WI) }\end{array}$ & $\begin{array}{l}\qquad W I=\operatorname{Ln}\left(\frac{A s}{\tan (S)}\right) \\
\text { As y S se definen igual al caso anterior }\end{array}$ & (5) & [35] \\
\hline $\begin{array}{l}\text { Índice de } \\
\text { erosión } \\
\text { potencial a } \\
\text { corto plazo } \\
\text { (TSP, USP, } \\
\text { SSP) }\end{array}$ & $\begin{array}{c}E I_{p}=k \sum\left(A_{p} P\right)^{m} S^{n} \\
A_{p} \text { es el área de acumulación de flujo } \\
\text { pesada por las precipitaciones }(\mathrm{P}) \text { para } \\
\text { cada píxel. } K \text { es el factor de erodabilidad, } \\
\text { se asume en esta investigación } k=1 \text {. Si } \\
m=n=1 \text {, se obtiene la potencia total de } \\
\text { flujo (TSP) Si } m \text { Si } m=1 / 3 \text { y } n=2 / 3 \text { se } \\
\text { obtiene la potencia por poder de cizalla } \\
\text { (SSP) = } 1 / 2 \text { y } n=1 \text {, se obtiene la potencia } \\
\text { por unidad del ancho del canal (USP) }\end{array}$ & (6) & [36] \\
\hline $\begin{array}{l}\text { Índice de } \\
\text { gradiente } \\
\text { longitudinal } \\
\text { del canal (SL) }\end{array}$ & $\begin{array}{l}\qquad S L=\frac{(\Delta H * L)}{\Delta L} \\
\Delta H \text { es el cambio en la elevación de la } \\
\text { sección, } \Delta L \text { es la longitud de ésta, } \mathrm{Y} L \text { es } \\
\text { la longitud total desde el punto medio de } \\
\text { la sección de interés hasta el punto más } \\
\text { alto del canal aguas arriba. }\end{array}$ & (7) & [37] \\
\hline $\begin{array}{l}\text { Índice de } \\
\text { empina } \\
\text { miento } \\
\text { (ksn) }\end{array}$ & $\begin{array}{l}\qquad k s n=\frac{S}{A^{-\theta}} \\
\text { Ksn y } \theta \text { son el índice de empinamiento, } \\
\text { y la concavidad, respectivamente. } \\
\text { Esté índice fue calculado usando la } \\
\text { herramienta TopoToolBox de Matlab, } \\
\text { para ello es necesario determinar el área } \\
\text { de acumulación de flujo tomando en } \\
\text { cuenta el patrón de precipitaciones de la } \\
\text { zona, y la pendiente a partir del modelo } \\
\text { de elevación digital }\end{array}$ & (8) & $\begin{array}{l}{[38,39,} \\
40,41]\end{array}$ \\
\hline
\end{tabular}

Con la base de datos de sismicidad se calcularon: energía, deformación y levantamiento sísmico denotados como ES, DS y $U$, respectivamente (Tabla 6). Con la base de datos de isótopos cosmogénicos [19] se obtuvo las tasas de erosión actual calculada por isotopos cosmogénicos.

Tabla 6. Parámetros sísmicos calculados para la zona de estudio a partir de la base de datos de eventos sismológicos reportados entre 1974 y 2020. 
Daniel R. Hernández Chaparro Mauricio A. Bermúdez Greg Hoke Helbert García Delgado Silvia Machuca

\begin{tabular}{|c|c|c|c|}
\hline Parámetro & Ecuación & & Referencia \\
\hline \multicolumn{4}{|c|}{$\log _{10}(E s)=b M_{i}+a$} \\
\hline $\begin{array}{l}\text { Energía sísmica } \\
\text { (Es) }\end{array}$ & $\begin{array}{l}\text { Los parámetros a y b de esta relación } \\
\text { se calculan mediante regresión lineal } \\
\text { de la relación magnitud-frecuencia } \\
\text { de los sismos. El área de dividió en } 4 \\
\text { y } 16 \text { cuadrantes con el propósito de } \\
\text { analizar su variabilidad espacial. M es } \\
\text { la magnitud en escala de Richter }\end{array}$ & (9) & {$[42]$} \\
\hline $\begin{array}{c}\text { Deformación } \\
\text { sísmica } \\
\text { horizontal } \\
\left(D S_{H}\right)\end{array}$ & $\begin{array}{l}D S=\left(\frac{1}{2 \mu \Delta V \Delta t}\right)\left(\frac{11^{a+9+9,1}}{1,5-b}\right)\left(10^{\left.(1,-b-b) M_{\max }\right)}\right. \\
\mathrm{M}_{\max } \text { es el máximo de la magnitud } \\
\text { observada, } \mu \text { es el módulo Young, } \Delta V \\
\text { representa el volumen de la corteza, } \\
\Delta t \text { es el periodo de tiempo de la base } \\
\text { de datos sismológicos }\end{array}$ & (10) & {$[43,3]$} \\
\hline \multirow[b]{2}{*}{$\begin{array}{c}\text { Deformación } \\
\text { sísmica vertical } \\
\left(\mathrm{DS}_{\mathrm{v}}\right)\end{array}$} & $D S_{v}=-D S_{H} \times 2,5 M a$ & \multirow[b]{2}{*}{ (11) } & \multirow[b]{2}{*}{ [43] } \\
\hline & $\begin{array}{c}\text { Se calcula durante los últimos } \\
2,5 \text { Ma para lograr extrapolar el } \\
\text { levantamiento sísmico en el tiempo } \\
\text { y hacerlos coincidir temporalmente } \\
\text { con los datos termocronológicos }\end{array}$ & & \\
\hline \multicolumn{4}{|c|}{$\boldsymbol{u}=\boldsymbol{h}_{\varepsilon} D S_{v}\left(\mathbf{1}-\frac{\boldsymbol{\rho}_{\varepsilon}}{\boldsymbol{\rho}_{m}}\right)$} \\
\hline $\begin{array}{l}\text { Levantamiento } \\
\text { sísmico }(u)\end{array}$ & $\begin{array}{l}h_{c} \text { es el espesor de la corteza tomado } \\
\text { del modelo CRUST } 1.0 \text { mencionado } \\
\text { anteriormente, } \rho_{c} \text { y } \rho_{m} \text { son los } \\
\text { promedios de las densidades de la } \\
\text { corteza y el manto, respectivamente }\end{array}$ & $(12)$ & {$[43,22,23]$} \\
\hline
\end{tabular}

A partir de los datos termocronológicos se calcularon las tasas de erosión a largo plazo mediante el uso de los códigos: PECUBE [4, 6] y Age2Edot [21] Finalmente, para hallar relaciones significativas entre estos parámetros, y facilitar la discusión, se realizó un análisis de correlaciones empleando los coeficientes de Pearson con un valor $p$ de 0,2 seguido de un análisis de regresión múltiple [44].

\section{RESULTADOS}

\subsection{Tasa de precipitaciones, Elevación, y Relieve local}

El promedio anual de precipitación ha fluctuado en la zona de estudio en las últimas décadas desde $3 \mathrm{~m} /$ año hasta $7 \mathrm{~m} /$ año; localmente, los bloques surorientales tienen valores de precipitación más altos ( $5 \pm 1 \mathrm{~m} / \mathrm{año}$ ) que el resto del área de estudio, siendo los bloques de Servitá y Guayuriba los que más reciben precipitación con un promedio anual de $6 \mathrm{~m} / a n ̃ o$ y $6,9 \mathrm{~m} / \mathrm{año}$, respectivamente, a diferencia de los bloques noroccidentales como Teusacá, Quetame y Chingaza que no superan el promedio de precipitación anual de 2,8 $\pm 0,6 \mathrm{~m} /$ año.
En cuanto a la distribución de elevaciones, éstas se encuentran en el rango comprendido entre 400 y $4000 \mathrm{msnm}$. Adicionalmente, el relieve de la zona se caracteriza por tener valores mayores a los 1000 metros. La Figura 10, muestra los mapas de relieve calculados a $1 \mathrm{~km}$ y 2,3 km, respectivamente. En cuanto a los bloques litotectónicos discriminados, se observa que el bloque Guayabetal presenta el valor máximo de relieve (2342 m) usando un radio de $1 \mathrm{~km}$, seguido del bloque Guatiquía. Por su parte, el promedio del relieve en toda la zona es de 1970 m, lo cual habla de una topografía abrupta en casi toda el área, donde el bloque de Servitá es el que presenta menor promedio de relieve con $1477 \mathrm{~m}$.

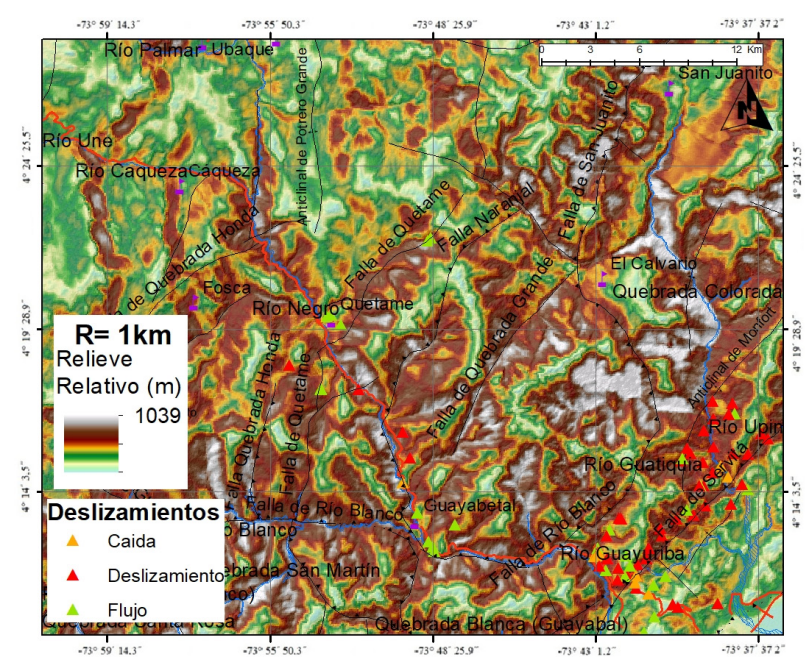

Figura 10. Mapas de relieve relativo calculado para un radio de $1 \mathrm{~km}$. Se reportan sobre estos los deslizamientos o fenómenos de remoción en masa descargados del catálogo electrónico del Sistema de Información de Movimientos en Masa (SIMMA, 2020). Sobre estos mapas se han colocado las principales fallas de la zona de estudio [7].

\subsection{Integral hipsométrica}

La hipsometría del área corresponde a un paisaje muy erosionado en general, donde en la Figura 12A se observa áreas en estado de desequilibrio ( $\mathrm{Hi}>0,6)$ en naranja y rojo.

\subsection{Atributos primarios y secundarios del terreno} Los atributos del terreno se presentan en la Figura 12. 
En particular, el índice de capacidad de transporte de sedimentos (STI) se visualiza en la Figura 11 (B). El índice de humedad topográfica (WI) se presenta en la Figura 11 (C), en la cual los sitios muy planos coinciden con las partes más húmedas del paisaje, tal como el sureste del área. El valor máximo del índice topográfico de humedad (WI) se presenta en el bloque de Servitá con un valor de 3,17 debido a su bajo relieve, el promedio en la zona es de 2,2 para el WI; mientras el índice STI presenta valores negativos y positivos asociado con zonas de sedimentación y erosión respectivamente, con valores positivos mayores para el bloque de Guayuriba de 0,045, lo que sugiere que este bloque, el cual se encuentra en el sector Chirajara, tiene un alto potencial erosivo y coincide con importantes deslizamientos en la zona.

Los índices de erosión potencial a corto plazo (EI), como el poder erosivo por cizalla (SSP, Shear Stress Power), se visualizan en la Figura 11 (D), el poder erosivo unitario (USP, Unit Stream Power) en la Figura 11(E) y el poder erosivo total (TSP, Total Stream Power) en la Figura 11 (F). El índice TSP presenta un promedio muy alto de 2,23E7 en el bloque Guayuriba, comparado con el promedio de todos los bloques de 3,6E6. Este comportamiento persiste con el índice USP, donde el bloque Guayuriba presenta el valor promedio de 685 que sobresale entre el promedio de todos los bloques de 275. También en el índice SSP, el bloque Guayuriba exhibe un valor alto de 54, siendo la mayor magnitud de este índice en los bloques donde se tiene una media de 32, siendo el bloque Guayuriba el que más presenta potencial erosivo a corto plazo, lo cual se debe a la confluencia del Río Negro y el Río Blanco en este bloque, por lo cual se concentra el alto poder erosivo en el Río Guayuriba. Después del bloque Guayuriba, el bloque Guatiquía exhibe un alto potencial erosivo en el índice USP y TSP con valores de 290 y 2,85E6, respectivamente.
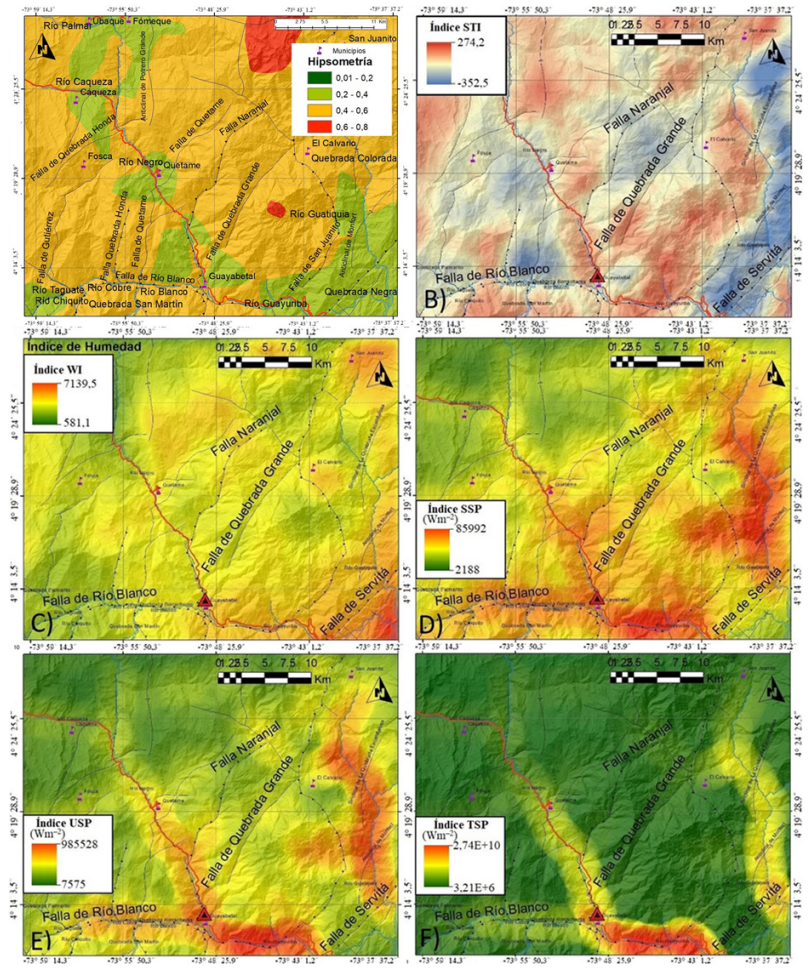

Figura 11. A) Mapa de valores de la integral hipsométrica en la zona de estudio, realizados con la herramienta TAK (Forte et al., 2019); B) Índice de transporte de sedimentos (STI); C) Índice Topográfico de Humedad (WI); D) Índice de erosión a corto plazo por poder de cizalla (SSP) (Wm2); E) Índice de erosión a corto plazo unitario por ancho del canal (USP) (Wm-2); F) Índice de erosión a corto plazo total (TSP) (Wm-2). Sobre cada uno de los mapas se muestran las principales fallas [7].

\subsection{Indice del gradiente longitudinal del canal (SL) y empinamiento ( $k s n$ )}

El índice SL se presenta en la Figura 12, para diferentes segmentos de perfiles longitudinales del Río Guayuriba. Además, se muestra el intervalo de precipitación y la litología presente en cada segmento. El índice SL presenta su valor máximo de 1321 en el bloque Guayuriba seguido del bloque de Río Blanco y Guayabetal, con 1112 y 939 respectivamente, siendo el promedio del índice $S L$ en todos los bloques de 709 . 

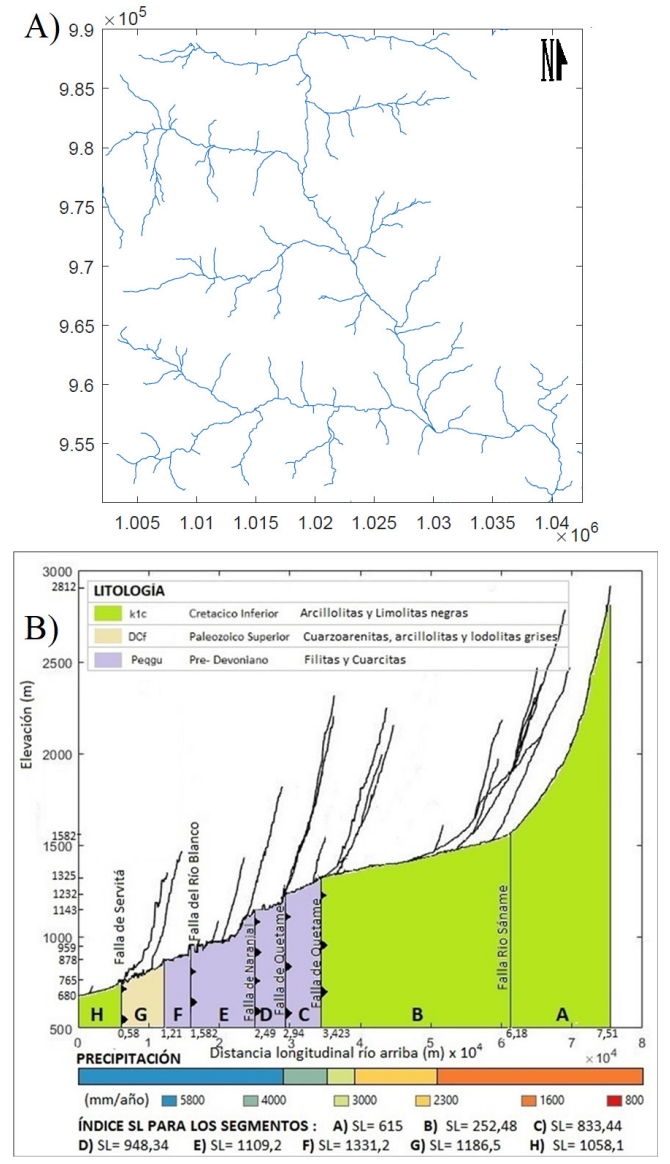

Figura 12. A) Red de drenajes de la cuenca del Río Guayuriba; B) Perfil del flujo para el río Guayuriba con valores del índice SL para cada segmento entre dos knickpoints, en el cual se interpretó visualmente colocando en yuxtaposición litologías, presencia de fallas y valores de precipitación media anual. Para la elaboración de esta figura se consideró el cambio de las tasas de precipitación con la altura y la distribución de fallas existentes en la zona [7].

En la Figura 13, se presenta el mapa con valores del índice ksn, Se observa en esta figura los sitios de muestreo con edades termocronológicas existentes. El índice ksn presenta una media de 243 para toda la zona siendo un valor alto de incisión, mientras el valor mínimo lo presenta el bloque de Servitá con 66.

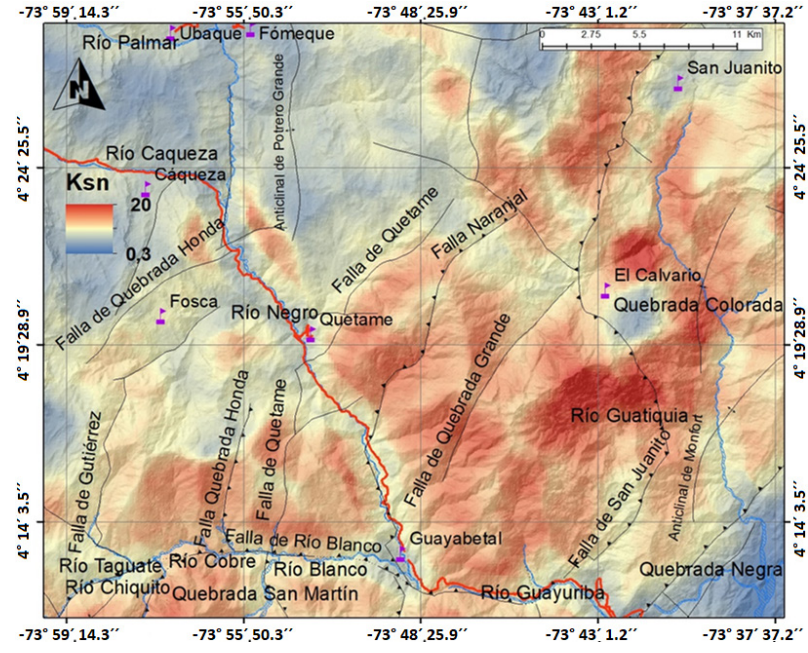

Figura 13. Mapa de índice de empinamiento (ksn). En este mapa se muestran las principales fallas de la zona [7].

\subsection{Parámetros sismológicos (energía, deformación y levantamiento)}

En los bloques litotectónicos de Naranjal y Guayabetal se registra la mayor actividad sísmica de la zona. En la Figura 14, se muestran los eventos sísmicos desde 1974 hasta el 2020 y los parámetros sísmicos calculados a partir de esa base de datos. En términos de la subdivisión en 4 y 16 cuadrantes, respectivamente, no introduce diferencias significativas en los valores b calculados. Sin embargo, debido a que la subdivisión está también relacionada con la escala de los fenómenos tectónicos observados, se decidió a lo largo de la investigación mantener ambas subdivisiones. La mayor deformación sísmica y levantamiento sísmico ocurre en la zona delimitada entre las fallas Naranjal y Quebrada Grande (Figuras 14 A y B). La energía sísmica liberada es mayor en el norte de la zona de estudio (Figuras 14 C y D).

La energía sísmica varía entre 1,18E5 J a 2,41E6 J para todos los bloques, y el valor promedio es $5 \mathrm{E} 5 \mathrm{~J}$. El valor máximo se encuentra en el bloque de Guayabetal 2,41E6 $J$, concentrándose la energía sísmica en este sector. Le siguen los bloques Quetame y Servitá con energía sísmica promedio de 5,545 J y 4,21E5 J, respectivamente. Se observa que estos bloques presentan los valores máximos de energía sísmica debido a que sus márgenes 
corresponden con las principales estructuras del área como las fallas de Servitá, Quetame y Naranjal. El resto de los bloques presentan energía sísmica baja de 2,5E5 $\pm 0,6 \mathrm{E} 5 \mathrm{~J}$, comparado con los bloques de Guayabetal, Quetame y Servitá.

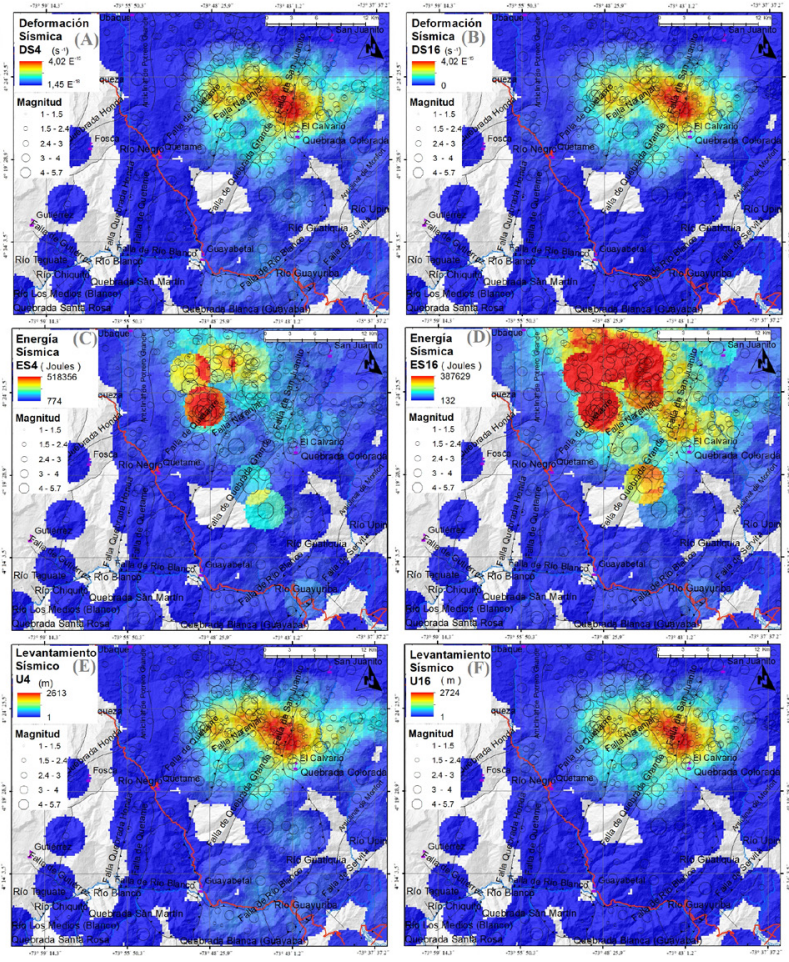

Figura 14. Parámetros sísmicos obtenidos al dividir la zona de estudio en 4 y 16 cuadrantes, respectivamente: (A) Deformación sísmica (DS4) y (B) Deformación sísmica (DS16). (C) Energía sísmica (ES4) y (D) Energía sísmica (ES16). (E) Levantamiento sísmico (LS4) y (F) Levantamiento sísmico (LS16), respectivamente. Se muestran las principales fallas geológicas de la zona [7].

4.6. Índice de erosión actual por datación de isótopos cosmogénicos (10Be).

En las terrazas del Río Guayuriba, existen 7 estimaciones de tasas de incisión (01, 03-08; Figura 11) previamente determinadas [19] a partir de mediciones de Berilio-10, las cuales fueron interpoladas en esta investigación, obteniéndose tasas de $\sim 75 \mathrm{~mm} / \mathrm{ka}$ (muestras 01, 03, 04, 08; Figura 15), mientras que las tasas de erosión aumentan significativamente a valores de 100, 479 y 670 $\mathrm{mm} / \mathrm{ka}(07,05$ y 06$)$ hacia el sur de la zona de estudio, respectivamente.

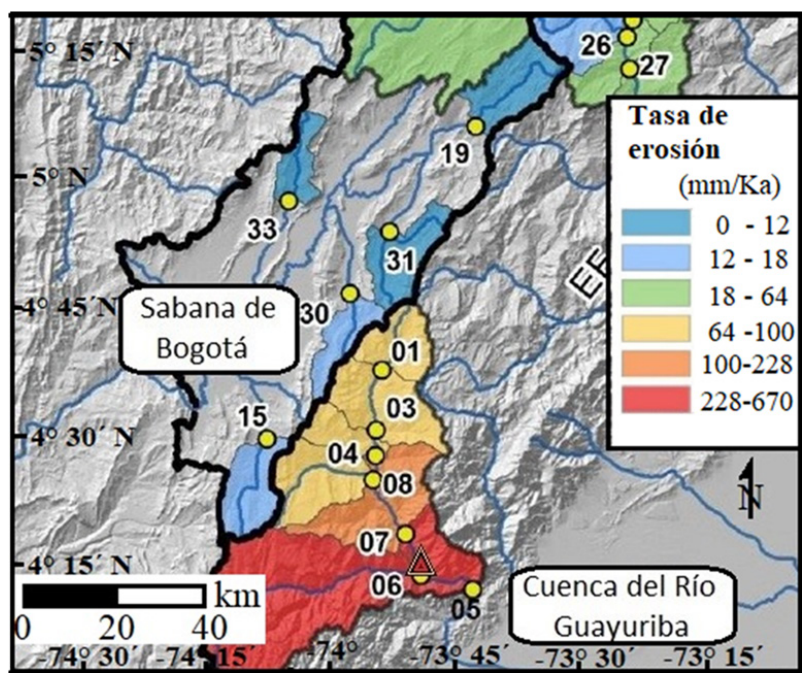

Figura 15. Distribución de tasas de erosión derivadas a partir de isótopos cosmogénicos (10Be), donde el triángulo rojo-amarillo corresponde al deslizamiento del km 58. Los números enteros corresponden a muestras en terrazas fluviales de distintas cuencas de la cordillera oriental (Modificado [19]).

\subsection{Tasa de erosión a largo plazo}

La tasa de erosión a largo plazo promedio para toda el área es de 2,37 mm/año, siendo una tasa muy alta de erosión, donde los valores más altos se concentran al oriente, en el bloque Guatiquía, con una tasa de 3,47

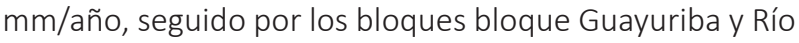
Blanco con una tasa de 2,97 mm/año y 2,63 mm/ año, respectivamente.

\subsection{Resultados del modelo directo termocinemático}

Las predicciones del modelo termocinemático directo de menor misfit $(3,327)$ son mostradas en la Figura 16. Los parámetros de entrada como se mencionó anteriormente, fueron temperatura basal de $400^{\circ} \mathrm{C}$ y la producción de calor de 1,2 mW/m2. Se consideró además una topografía en equilibrio para simplificar el tiempo de cómputo. 


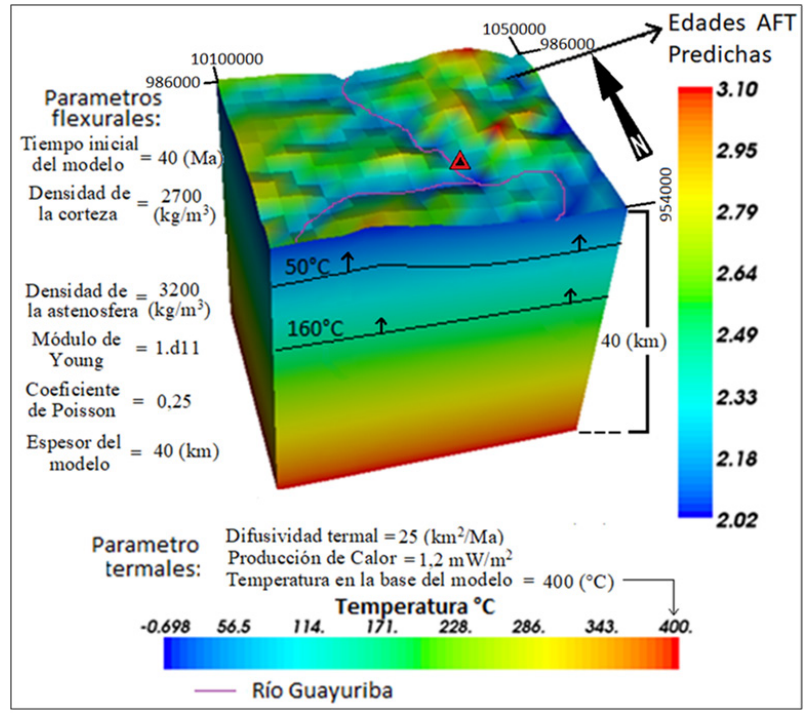

Figura 16. Edades AFT predichas por el modelo directo termocinemático 3D de menor misfit $(3,327)$.

La Figura 17, resume la variación de las tasas de exhumación predichas para el modelo de menor misfit. Considerando tres fases de exhumación, se obtuvo el primer pulso entre $40 \mathrm{Ma}$ y $25 \mathrm{Ma}$ con una tasa de exhumación de 0,5 km/Ma. Luego en el segundo pulso entre 25 Ma y $15 \mathrm{Ma}$, la tasa de exhumación decrece a 0,1 km/Ma, y a partir de los 15 Ma hasta el presente, la tasa de exhumación aumenta abruptamente a $2 \mathrm{~km} / \mathrm{Ma}$. Las edades predichas se convirtieron a tasas de exhumación a largo plazo, las cuales están resumidas en la tabla 6.

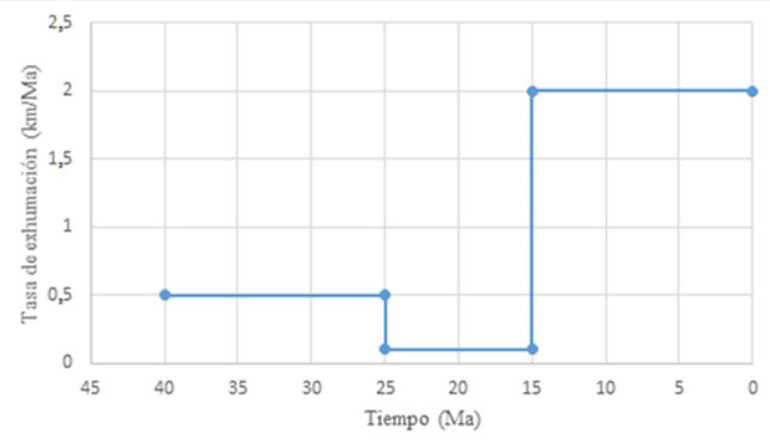

Figura 17. El gráfico ilustra las variaciones de las tasas de exhumación en el tiempo para el modelo de menor misfit $(3,327)$.
Se presenta en la Figura 18, la comparación de perfiles edad-elevación sintéticos versus observados.

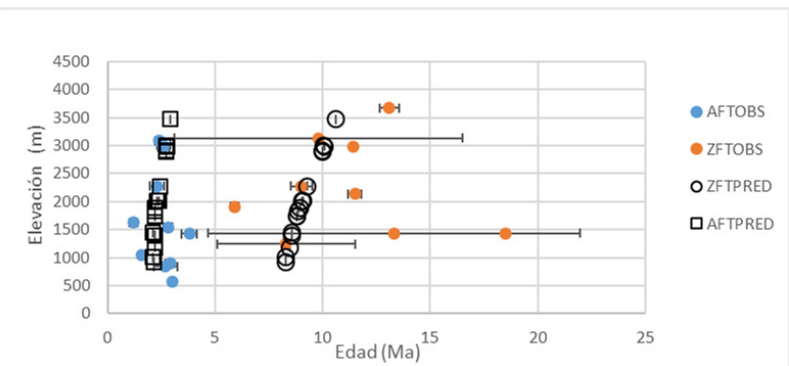

Figura 18. Comparación de relaciones edad-elevación observados (azul: AFT y naranja: ZFT) y predicho (cuadrados: AFT y círculos: ZFT) para el modelo de menor misfit $(3,327)$.

Para la Figura 18, se realizó una regresión lineal con el propósito de obtener estimaciones de tasas de exhumación de los dos sistemas termocronológicos utilizados, obteniéndose valores de coeficientes de determinación: R2=0,9194 y R2=0,9856, para las edades AFT y ZFT, respectivamente. Adicionalmente, se obtuvieron las siguientes expresiones:

$$
\begin{aligned}
& \text { Elevación }[m]=2663,4\left[\frac{m}{M a}\right] * \boldsymbol{F T} \boldsymbol{A}[M a]-4321,1[\mathrm{~m}] \\
& \text { Elevación }[\mathrm{m}]=1058,6\left[\frac{\mathrm{m}}{M a}\right] * \boldsymbol{F T Z}[\mathrm{Ma}]-7657,4[\mathrm{~m}]
\end{aligned}
$$

La tasa de erosión derivada de las relaciones edad AFT versus elevación es 2,6 km/Ma; mientras que la tasa de erosión estimada de las relaciones edad ZFT versus elevación es de $1 \mathrm{~km} / \mathrm{Ma}$. Tal diferencia entre ambas estimaciones sugiere que la velocidad de exhumación se ha incrementado desde los últimos 5 Ma. La Figura 19A muestra las edades de AFT predichas por el código PECUBE para toda la zona de estudio, y en más detalle las del Bloque Guayabetal en la Figura 19B, en el cual coinciden las mayores edades de AFT predichas con la deformación sísmica. 

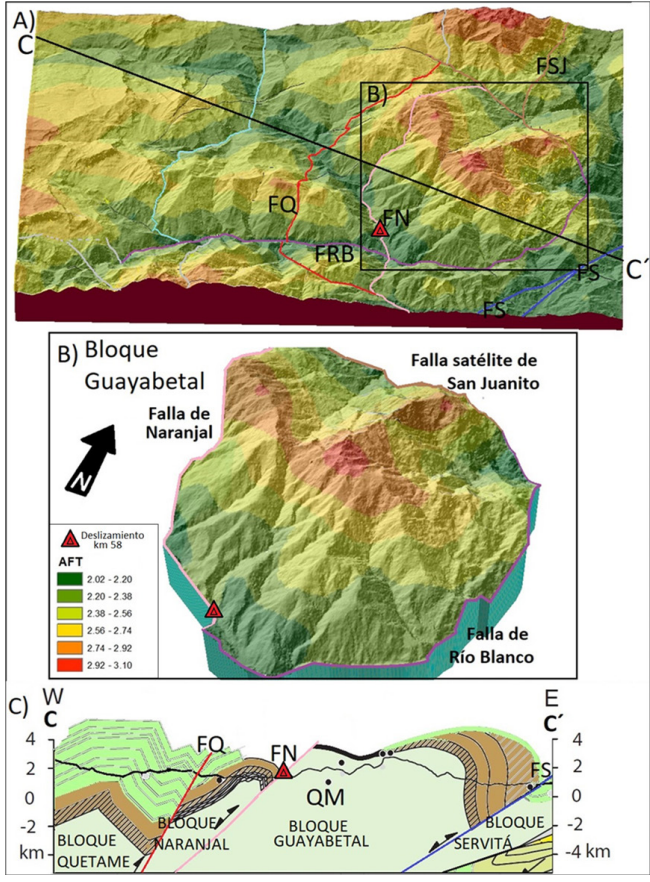

Figura 19. A) Edades AFT predichas por PECUBE para la zona de estudio. Las líneas de color son las fallas en el área: FSJ= Falla de San Juanito, FN= Falla de Naranjal, $\mathrm{FQ}=$ Falla de Quetame, FRB= Falla de Río Blanco, FS= Falla de Servitá [7]; B) Edades AFT predichas en el Bloque de Guayabetal; C) Perfil transversal de la zona de Estudio (Modificada [8]).

Finalmente, para facilitar la discusión, se presentan en la Tabla 7 los valores promedios de los parámetros geomorfológicos y sísmicos de cada uno de los bloques litotectónicos de la Figura 9.

Tabla 7. Valores promedios de control potencial para cada bloque litotectónico propuesto: ES = Energía Sísmica (Joules); DS = Tasa de deformación sísmica (s-1); $U=$ Levantamiento sísmico $(\mathrm{m}) ; \mathrm{P}=$ Precipitación (m/año) para el período 1990-2019; STI = Índice de transporte de sedimentos; $W I$ = Índice de humedad; TSP = Poder de flujo total (Wm-2); USP = Poder de flujo unitario (Wm$2) ; \mathrm{SSP}=$ Poder de flujo por cizalla $(\mathrm{Wm}-2) ; \mathrm{SL}=$ Índice del gradiente longitudinal del canal $(\mathrm{m}) ; \mathrm{ksn}=$ Índice de inclinación; $\mathrm{HI}=$ Integral Hipsométrica; R1 = Relieve radio 1-km (m); erAFT = Erosión a largo plazo observada para datos AFT, er-CN = Erosión por datación de isótopos cosmogénicos ( $\mathrm{mm} / \mathrm{ka})$.

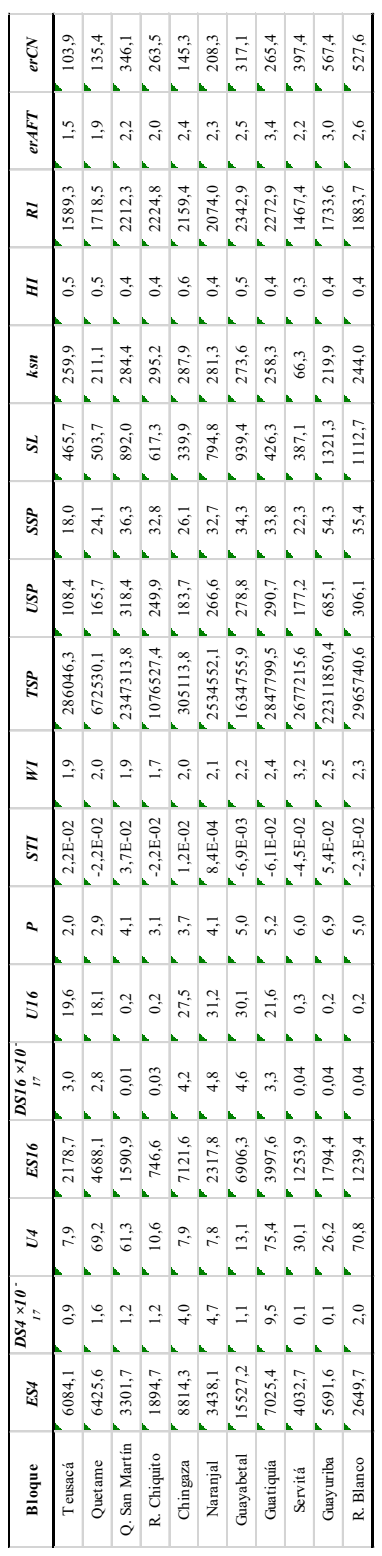

\section{DISCUSIÓN}

Los Himalayas, Los Andes y los orógenos de Nueva Zelanda constituyen regiones claves donde las interacciones entre tectónica y aspectos climáticos, en particular el patrón de precipitaciones, han sido documentadas, las cuales pueden influenciar de manera fundamental la evolución 
Daniel R. Hernández Chaparro Mauricio A. Bermúdez Greg Hoke Helbert García Delgado Silvia Machuca

individual del relieve [45, 46]. Para el área de estudio estas relaciones son desconocidas. Con el propósito de facilitar la discusión, trataremos de dar respuestas a preguntas claves como, por ejemplo: ¿Cómo influye la sismicidad en los bloques con más deslizamientos (bloques surorientales)? ¿Qué bloques presentan correlaciones altas entre relieve y clima o relieve y tectónica? ¿Existirá acople entre los procesos climáticos y tectónicos?, si esto es así ¿En qué escala de tiempo se presentan tales interacciones?

Con los resultados de la Tabla 7, se realizó un análisis de correlaciones de Pearson, presentado bajo la forma de correlogramas y mostrado en la Figura 20. En la Figura 20A, se incorporan todos los bloques considerados, mientras que en la Figura 16B se consideraron sólo los bloques litotectónicos con mayor actividad sísmica cercanos a la zona de deslizamiento (Naranjal, Guayabetal, Guatiquía, Servitá, Guayuriba y R. Blanco). El incremento en los valores de la excentricidad (relaciones entre eje mayor y eje menor) de la elipse sugiere una mayor dispersión entre los pares de variables que están siendo comparados. En ambos correlogramas, las relaciones entre todos los parámetros sísmicos ES4, DS4, ES16, DS16, U4 y U16 son esperadas ya que son calculadas de la misma forma, por lo que son descartadas. Análogamente, las correlaciones entre las variables P, TSP, SSP, USP, WI, y STI tampoco deben ser consideradas porque todos los índices de erosión a corto plazo fueron pesados por las precipitaciones.

La Figura 20A muestra importantes relaciones $(r=0,7)$ entre la deformación sísmica calculada para toda el área dividida en cuatro cuadrantes DS4 y las tasas de erosión erAFT y erCN. Sin embargo, cuando se refina la escala incluyendo procesos locales, no se observan correlaciones entre DS16 y las tasas de erosión antes mencionadas. En contraste, correlaciones entre el patrón de precipitaciones $\mathrm{P}$ y las tasas de erosión erAFT y erCN soportaría los efectos de un control climático; la correlación entre P y erCN $(r=0,8)$ es ligeramente mayor que la de P y erAFT $(r=0,7)$. Todos los índices de erosión a corto plazo TSP, USP y SSP correlacionan con ambas tasas de erosión $(r \geq 0,5)$. Sin embargo, los mayores valores de $r$ se observan en las relaciones entre TSP, USP, SSP y erCN ( $r$ $\geq 0,7)$. La correlación entre ksn y R1 $(r=0,7)$, sugiere que el relieve actual está siendo afectado por los procesos de incisión de ríos; pero a su vez, la pérdida de correlación entre R1 y las tasas de erosión erAFT y erCN $(r \leq 0,4)$, al igual que R1 y $\mathrm{P}(r<0)$, sugieren que al considerar todos los bloques no se puede discriminar controles tectónicos o climáticos sobre el relieve.

En contraste, la Figura 20B, para los bloques con mayor actividad sísmica, refleja una importante relación entre R1 y ES4 ( $r=0,6), R 1$ y ES16 ( $r=0,8)$, R1 y DS16 ( $r=0,8)$ y U16 ( $r=0,8)$. Para estas zonas, la correlación entre relieve $\mathrm{R} 1$ y $\mathrm{P}$ es negativa $(r=-0,6)$. Con respecto a las tasas de erosión, se observa que localmente la tectónica no pareciera controlar las tasas de erosión obtenidas de las edades AFT ya que no existe correlación entre ES16, DS16 y U16 con erAFT. Sin embargo, la correlación entre U4 y erAFT $(r=0,6)$ sugiere que globalmente la tectónica tiene una influencia importante sobre las tasas de erosión derivada de las edades AFT y sobre el relieve. Observando las correlaciones entre P y erCN $(r=0,7)$, y los índices TSP con erCN $(r=0,6)$ y USP versus erCN $(r=0,6)$ sugiere un desacople entre aspectos climáticos y tectónicos. A partir de cierto momento, la precipitación pareciera controlar las tasas de erosión más recientes.

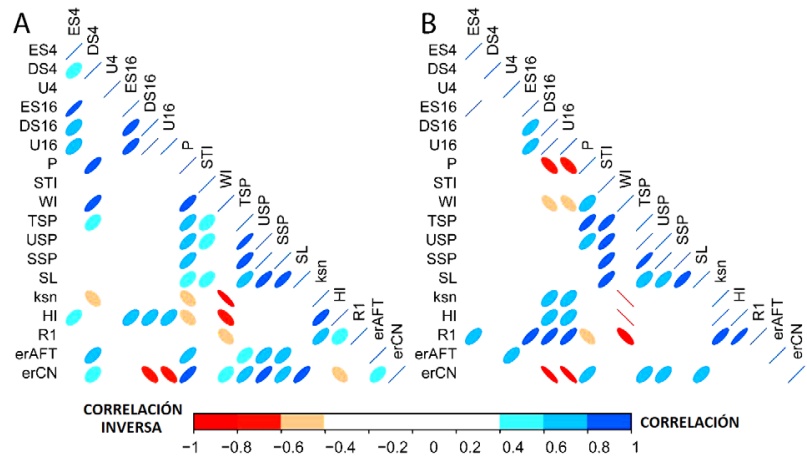

Figura 20. Correlogramas (A) para todos los bloques litotectónicos considerados, (B) sólo los cercanos a la zona de deslizamientos;

El análisis realizado sugiere que la morfología actual del área de estudio no es afectada uniformemente por la 
tectónica y las precipitaciones. Se pueden distinguir dos áreas distintas de control, la primera localizada en al noroccidente (Bloques de Teusacá, Chingaza y Quetame; Figura 1B) con tasas bajas de erosión y actividad tectónica donde la evolución del paisaje es más pasiva, y el relieve correlaciona fuertemente con la precipitación, siendo ésta el principal factor que controla esta área noroccidental. Por otra parte, la relación de la precipitación con las tasas de erosión a corto y a largo plazo en toda la zona, sugieren el control del clima en el rejuvenecimiento de la superficie.

La segunda zona se encuentra en el macizo de Quetame y en el piedemonte llanero, representado por los bloques surorientales (Guatiquía, Guayabetal, Guayuriba, Naranjal, Servitá y Río Blanco). En estas zonas es donde ocurrió el deslizamiento que motivó el presente estudio y posiblemente exista una concentración de actividad sísmica a lo largo del tiempo donde se evidencia el aporte tectónico para la formación de estos bloques, sugerido por la relación del relieve con los parámetros sísmicos (DS16 y U16). Sin embargo, esta observación debe ser tomada con cautela ya que la ventana de tiempo observado es menor a 50 años. Aunque el control tectónico es significativo, recientemente el clima ha rejuvenecido el paisaje, posiblemente desde el Pleistoceno. Una vez que se produce la rápida exhumación ( $2 \mathrm{~km} / \mathrm{Ma}$ ) del macizo de Quetame (Parra et al., 2009), se genera una barrera orográfica en la cual los vientos húmedos provenientes del Amazonas se concentran, lo cual explicaría por qué los termocronómetros muy someros, cómo en el caso de los isótopos cosmogénicos por 10Be (erCN), correlacionan con la variable precipitación.

Relación entre interacciones clima-tectónica en el deslizamiento del km 58

Para el deslizamiento del km 58 presente el bloque litotectónico de Guayabetal, un alto grado de criticidad resulta cuando se combinan altas susceptibilidades en esta zona suroriental del área de estudio, debida a factores topográficos (geomorfología abrupta producida por la concentración de deformaciones en el flanco oriental), geológicos (la litología de terrazas aluviales colgadas con alta disección sobre filitas fracturadas), climáticos (precipitación muy alta con más de $7 \mathrm{~m} /$ año) y sísmicos (la falla de Naranjal) influye de manera directa en el deslizamiento al estar a menos de $1 \mathrm{~km}$ de la zona. Observando el catálogo sísmico se discriminó un evento en Quetame de magnitud 1,8 y profundidad de 1,4 km el 13 de junio del 2019, esto coincidió con el día que se inició el movimiento en el deslizamiento de estudio, asumiendo por lo tanto que la trasferencia de la deformación cosísmica se hizo a través de dicha falla transfiriendo cargas vibratorias las cuales producen un aumento del esfuerzo cortante y aumento de la presión de poros, creando estos aumentos una disminución en la resistencia por acción de las deformaciones las cuales disminuyen notablemente el factor de seguridad del talud.

En este caso, el deslizamiento complejo en su mayoría de tipo traslacional ha sufrido disección alta en el talud desde el primer movimiento antes mencionado, quedó expuesto al recibir una mayor erosión, reactivando la zona y generando una zona de alto riesgo. Hasta el momento, la erosión de la ladera no había sido considerada la compleja interacción entre clima-tectónica y exhumación de bloques litotectónicos no se había tomado en cuenta. Una importante reactivación de fallas en el bloque Guayabetal posiblemente transfirió la deformación cosísmica por la falla de Naranjal hasta el lugar del deslizamiento donde la litología (filitas cuarzosas fracturadas y sobre ellas terrazas coluvio-aluviales colgadas) amplificarían la onda sísmica.

\section{CONCLUSIONES Y RECOMENDACIONES}

El modelado directo termocinemático 3D permitió establecer la historia de exhumación de este sector de la Cordillera Oriental, los tres pulsos y sus velocidades, corroborando la hipótesis de levantamiento rápido conducido principalmente por la tectónica en el primer pulso entre $40 \mathrm{Ma}$ y $25 \mathrm{Ma}$ con una tasa etapa de exhumación lenta de 0,1 km/Ma, luego se aceleró esta exhumación en un segundo pulso ocurrido entre $25 \mathrm{Ma}$ a $15 \mathrm{Ma}$ con una velocidad 0,5 km/Ma controlada por 
la tectónica más que por el clima y, posteriormente un levantamiento reciente en el último y tercer pulso entre 15 Ma con tasas considerablemente altas (2 km/Ma), conducido por la interacción de clima y tectónica.

El principal factor dominante del paisaje hoy en día es la incisión fluvial primariamente impulsada por el incremento en la pendiente regional del orógeno por acumulación progresiva del acortamiento y engrosamiento cortical [19]. A nivel general la tectónica, ejercería un control importante sobre el relieve, pero su efecto disminuiría sobre las tasas de erosión a largo y corto plazo. Se toma un importante rol el clima para explicar las tasas de erosión.

La actividad tectónica puede estar creando topografía en zonas de alta sismicidad (Bloques Guayuriba, Río Blanco y Guayabetal), y en conjunción con el clima han incrementado la incisión fluvial del Rio Blanco y el Río Negro, ambos tributarios del Río Guayuriba.

La morfología actual del área de estudio no es afectada de la misma forma por la tectónica y el clima, se pueden distinguir dos áreas distintas, la primera localizada en al NW con tasas bajas de erosión y actividad tectónica donde la evolución del paisaje es más pasiva y donde el relieve correlaciona fuertemente con la precipitación, siendo el clima el principal factor que controla los bloques NW: Teusacá, Chingaza, Quetame. La segunda zona se encuentra desde macizo de Quetame hasta el piedemonte oriental, correspondiendo a los bloques SE; en esta zona se concentra de actividad sísmica con las principales estructuras presentes como las Fallas de Naranjal, Quetame, San Juanito y Servitá con alto potencial sismogénico. Las fuertes relaciones de los parámetros sísmicos con el relieve y el índice de incisión ksn sugieren que la tectónica controló la formación de esta zona controlando el acortamiento y engrosamiento del orógeno el cual incrementó la pendiente aumentando el poder erosivo de incisión (ksn) hasta el Pleistoceno, cuando el clima rejuvenece los bloques SE.

Un aspecto que no pudo ser evaluado en la zona es el impacto de las intervenciones antrópicas sobre el relieve, a futuro sería vital incluir esa información en este tipo de análisis.

Esta relación del efecto sísmico en la estabilidad de taludes relacionado con la constante interacción de factores operantes como la tectónica, clima y la exhumación de un determinado bloque desencadenando un fenómeno de remoción en masa de una magnitud e impacto nacional representa una contribución al conocimiento de las geociencias a la geología aplicada o la ingeniería geológica.

Se recomienda a futuro fechar mediante isótopos cosmogénicos las muestras seleccionadas en la terraza objeto de este estudio. Adicionalmente, sería ideal incorporar esas nuevas edades con el propósito de realizar modelos termocinemáticos inversos.

\section{AGRADECIMIENTOS}

A la Universidad Pedagógica y Tecnológica de Colombia (UPTC), Sogamoso Colombia, por el apoyo brindado durante el desarrollo de la presente investigación. Especialmente, por el financiamiento proporcionado a través del proyecto DIN-SGI-2666 de la Universidad Pedagógica y Tecnológica de Colombia (UPTC), y por el financiamiento proporcionado a través de las convocatorias de movilidad internacional que permitieron la estancia del Dr. Greg Hoke en la UPTC durante las campañas de muestreo de terrazas cercanas a la zona de estudio.

\section{REFERENCIAS}

[1] Bermúdez, M., van der Beek, P., Bernet, M. "Asynchronous Miocene-Pliocene exhumation of the central Venezuelan Andes," Geology, v. 39, p. 139-142, doi:10.1130/G31582.1, 2011.

[2] Bermúdez, M., van der Beek, P., Bernet, M. "Exhumación Diacrónica durante el MiocenoPlioceno de Los Andes de Mérida" Revista Tribuna del Investigador, Universidad Central de Venezuela, v. $12,(2)$, p. $20-23,2011$. 
[3] Bermúdez, M., van der Beek, P., Bernet, M. "Strong tectonic and weak climatic control on exhumation rates in the Venezuelan Andes" Lithosphere, The Geological Society of America. doi: 10.1130/L212.1. 2013.

[4] Braun, J. "PECUBE: A new finite-element code to solve the $3 \mathrm{D}$ heat transport equation including the effects of a time-varying, finite amplitude surface topography" Computers \& Geosciences, 29, 787794, 2003.

[5] Braun, J., van der Beek, P., Batt, G. "Quantitative Termochronology. Numerical Methods for the Interpretation of Thermochronological Data" Cambridge university. 272 p, 2006.

[6] Braun, J., van der Beek, P., Valla, P., Robert, X., Herman, F., Glotzbach, C., Pedersen, V., Perry, C., Simon-Labric, T., and Prigent, C. "Quantifying rates of landscape evolution and tectonic processes by thermochronology and numerical modeling of crustal heat transport using PECUBE" Tectonophysics, v. 524-525, p. 1-28, 2012.

[7] Gómez-Tapia, J., Montes, N.E., Nivia, Á., Diederix, H. “Mapa Geológico de Colombia 2015: Escala 1:100 000," Servicio Geológico Colombiano, Bogotá, 2 hojas, 2015.

[8] Mora, A., Parra, M., Strecker, M. R., Sobel, E. R., Hooghiemstra, H., Torres, V., and Vallejo-Jaramillo, J. "Climatic forcing of asymmetric orogenic evolution in the Eastern Cordillera of Colombia" Geol. Soc. Am. Bull., 120, 930 - 949, doi:10.1130/ B26186.1. 2008

[9] Parra, M., Mora, A., Sobel, E.R., Strecket, M., González, R. "Episodic orogenic front migration in the northern Andes: Constraints from lowtemperature thermochronology in the Eastern Cordillera, Colombia." Tectonics, 28, TC4004, doi:10.1029/2008TC002423. 2009.

[10] Cediel, F., Shaw, R. P., Cáceres, C. "Tectonic assembly of the Northern Andean block": 815-848. Retrieved from http://archives.datapages.com/data/ specpubs/memoir79/CHAPTER37/CHAPTER37. HTM 2003.

[11] Mora, A., Parra, M., Strecker, M. R., Kammer
A., Dimaté, C., and Rodriguez, F "Cenozoic contractional reactivation of Mesozoic extensional structures in the Eastern Cordillera of Colombia.\# Tectonics, 25, TC2010, doi:10.1029/ 2005 TC001854.

2006.

[12] Bakun, W. H., and Wentworth, C.M. "Estimating earthquake location and magnitude from seismic intensity data, Bull." Seism. Soc. Am., 87: 15021521. 1997.

[13] Salcedo, Elkin y Augusto Gómez. "Estudio macrosísmico del terremoto del 18 de octubre de 1743 en la región central de Colombia." Boletín de Geología 35 (1): 109-128. 2013.

[14] Guerrero, J. "A proposal on the classification of System Tracts: Application to the Allostratigraphy and Sequence Stratigraphy of the Cretaceous Colombian Basin Part 1: Berrriasian to Hauterivian": Geología Colombiana, No 27. Bogotá. 2002.

[15] Ulloa et al. "Caracterización de Unidades Geológicas y Geomorfológicas de Colombia - Formación Fómeque, Formación Lutitas de Macanal": INGEOMINAS, Bogotá. 2000.

[16] Renzoni, G., "Geología del Macizo de Quetame. Geología Colombiana", Universidad Nacional de Colombia, Facultad de Ciencias, Departamento de Geología, Bogotá. No. 5: p.75-127, 1968.

[17] Pulido, O., y Gómez, L. "Geología de la plancha 266 - Villavicencio (Informe y mapa a escala 1:100.000)": INGEOMINAS, Bogotá. 2001.

[18] Campbell, C.J., \& Burgl, H. "Section Through the Eastern Cordillera of Colombia, South America." Geol. Soc. Am. Bull., Vol. 76, 1965.

[19] Struth, L., Babault, J., Teixell, A. "Drainage reorganization during mountain building in the river system of the Eastern Cordillera of the Colombian Andes." Geomorphology, 250, 370383, doi: 10.1016/j.geomorph.2015.09.012. 2015.

[20] Perne, M., Covington, M. D., Thaler, E. A., \& Myre, J. M. "Steady state, erosional continuity, and the topography of landscapes developed in layered rocks." Earth Surf, Dynam., 5(1), 85-100. doi:10.5194/esurf-5-85-2017, 2017. 
Daniel R. Hernández Chaparro

Mauricio A. Bermúdez

Greg Hoke

Helbert García Delgado

Silvia Machuca

[21] Brandon, M.T., Roden-Tice, M.K., Garver, J.I. "Late Cenozoic exhumation of the Cascadia wedge in the Olympic Mountains northwest Washington state." Geological Society of America Bulletin 110, 985 1009, 1998.

[22] Bassin, C., Laske, G., Masters, G. "The Current Limits of Resolution for Surface Wave Tomography in North America." EOS Trans AGU, 81, F897bassin laskte, 2000.

[23] Laske, G., Masters., G., Ma, Z., Pasyanos, M., Update on CRUST1.0 - A 1-degree Global Model of Earth's Crust: Geophys. Res. Abstracts, 15, Abstract EGU2013-2658, 2013.

[24] Wagner G., y Van Den Haute P. "Fission-Track Dating": Dordrecht (Kluwer Academic Publishers). 285 pp. Mineralogical Magazine, 57(389), 760-761. doi:10.1180/minmag.1993.057.389.28 1992.

[25] Gómez, E., Jordan, T.E., Allmendinger, R.W., Cardozo, N. "Development of the Colombian forelandbasin system as a consequence of diachronous exhumation of the northern Andes." GSA Bulletin, v.117 (9), p.1272-1292, 2005.

[26] Braun, J., Robert, X. "Constraints on the rate of postorogenic erosional decay from low-temperature thermochronological data: application to the Dabie Shan, China." Earth Surface Processes and Landforms, v. 30, p. 1203-1225, 2005.

[27] Watson, D. F. y Philip, G. M. "A Refinement of Inverse Distance Weighted Interpolation" Geoprocessing 2:315-327, 1985

[28] UTGS, "Plan de Manejo y Ordenamiento de la Cuenca del Río Guayuriba, Unión temporal Guayuriba sostenible," Bogotá D. C. Recuperado de ftp://rioguayuriba\%7Canonymous@200.21.21.36/ Anexo_5_1_Tomo_3_Fase_Diagnostico/ Cap 4, pag 79-83, 2018.

[29] Clarín. "El epicentro fue en El Calvario, $50 \mathrm{~km}$. al sudeste De Bogotá," 25 de mayo del 2008, Clarín. Recuperado de: https://www.clarin.com/edicionesanteriores/terremoto-colombia-dejo-muertos_0_ SJ4GTz6RaFl.html 2008.

[30] SIMMA. "Sistema de Información de Movimientos en Masa." Bogotá, Dirección de Geoamenazas del Servicio Geológico Colombiano, catálogo de movimientos en masa (http://simma.sgc.gov.co/\#/, consultada en junio de 2020).

[31] Montgomery, D. R., \& Brandon, M. T. "Topographic controls on erosion rates in tectonically active mountain ranges." Earth and Planetary Science Letters, 201(3), 481-489. doi: https://doi. org/10.1016/S0012-821X(02)00725-2, 2002.

[32] Pike, R.J., Wilson, S.E. "Elevation-Relief Ratio, Hypsometric Integral and Geomorphic AreaAltitude Analysis." Geological Society of America Bulletin, 82, 1079-1084. http://dx.doi. org/10.1130/0016-7606(1971)82[1079:ERHIAG]2 $0 . C O ; 2,1971$

[33] Moore, I.D., Burch, G.J. "Physical Basis of the Length Slope Factor in the Universal Soil Loss Equation." Soil Science Society of America, 50, 1294-1298.

[34] Goulsbra, C., Evans, M., Lindsay, J. "Temporary streams in a peatland catchment: the pattern and timing of stream network expansion and contraction and controls on these." Geophys, Res. Abstr., 14, EGU2012-11014-1, 2012.

[35] Beven, K.J. Kirkby, M.J. "Un modelo de hidrología de cuenca de contribución variable con base física." Boletín de Ciencias Hidrológicas, 24 (1): 43-69. doi : 10.1080 / $02626667909491834,1979$.

[36] Tucker, G., y Slingerland, R. " Drainage basin responses to climate change", Water Resources research, Vol. 33, NO. 8, Pages 2031-2047, 1997.

[37] Hack, J. "Stream-profile analysis and streamgradient index." Journal of Research of the U.S, Geological Survey, p 421-429, 1973.

[38] Flint, J. "Stream gradient as a function of order magnitude and discharge." Water Resourse. Res. 10, 969-973, 1974.

[39] Whipple, K., y Tucker, G., "Dynamics of the streampower model: implications for the height limits of mountain ranges, landscape response timescales and research needs." J. Geophys. Res. 104, 1766117674, 1999.

[40] Schwanghart, W., Kuhn, N. J. "TopoToolbox: A set of Matlab functions for topographic analysis," Environ, Modell. Softw., 25, 770-781, https://doi. 
org/10.1016/j.envsoft.2009.12.002,. a, b, 2010.

[41] Schwanghart, W., Scherler, D. "Short Communication: TopoToolbox 2 - MATLAB-based software for topographic analysis and modeling in Earth surface sciences." Earth Surf, Dynam., 2, 1-7, https://doi.org/10.5194/esurf-2-1-2014,. a, b, 2014.

[42] Gutenberg, B., Richter, C.F. "Seismicity of the Earth and Associated Phenomena." Princeton, New Jersey, Princeton University Press, 245 p, 1954.

[43] Braun, J., Burbidge, D.R., Gesto, F.N., Sandiford, M., Gleadow, A.J.W., Kohn, B.P., Cummins, P.R. "Constraints on the current rate of deformation and surface uplift of the Australian continent from a new seismic database and low-T thermochronological data." Australian Journal of Earth Sciences, v. 56, p. 99-110, doi:10.1080/08120090802546977, 2009.

[44] Kendall, M.G., Stuart, A., "The Advanced Theory of Statistics," v. 2: Inference and Relationship: Griffin. ISBN 0-85264-215-6, 1973.

[45] Koons, P. O., Zeitler, P. K., Chamberlain, C. P., Craw, D., and Melzer, A. S., "Mechanical links between river erosion and metamorphism in Nanga Parbat, Pakistan Himalaya", Am. J. Sci., 302, 749- 773, doi:10.2475/ajs.302.9.749, 2002.

[46] Sobel, E.R., Hilley, G.E., Strecker, M.R. "Formation of internally drained contractional basins by aridity-limited bedrock incision." Journal of Geophysical Research. Solid Earth, 108, 2344, doi: 10.1029/2002JB001883, 2003. 